\title{
Star formation and black hole accretion activity in rich local clusters of galaxies ${ }^{\star}$
}

\author{
Matteo Bianconi ${ }^{1}$, Francine R. Marleau ${ }^{1}$, and Dario Fadda ${ }^{2,3}$ \\ ${ }^{1}$ Institut für Astro und Teilchenphysik, Leopold Franzens Universität Innsbruck, Technikerstraße 25/8, 6020 Innsbruck, Austria \\ e-mail: matteo.bianconi@uibk.ac.at \\ 2 Instituto de Astrofisica de Canarias, 38205 La Laguna, Tenerife, Spain \\ ${ }^{3}$ Universidad de La Laguna, Dpto. de Astrofísica, 38206 La Laguna, Tenerife, Spain
}

Received 30 July 2015 / Accepted 13 January 2016

\begin{abstract}
Context. We present a study of star formation and central black hole accretion activity of galaxies that are hosted in the two nearby $(z \sim 0.2)$ rich galaxy clusters Abell 983 and 1731

Aims. We aim to quantify both the obscured and unobscured star formation rates, as well as the presence of active galactic nuclei (AGN) as a function of the environment in which the galaxy is located.

Methods. We targeted the clusters with unprecedented deep infrared Spitzer observations ( $0.2 \mathrm{mJy}$ at 24 micron), near-IR Palomar imaging and optical WIYN spectroscopy. The extent of our observations ( $\sim 3$ virial radii) covers the vast range of possible environments, from the very dense cluster centre to the very rarefied cluster outskirts and accretion regions.

Results. The star-forming members of the two clusters present star formation rates that are comparable with those measured in coeval field galaxies. Analysis of the spatial arrangement of the spectroscopically confirmed members reveals an elongated distribution for A1731 with respect to the more uniform distribution of A983. The emerging picture is compatible with A983 being a fully evolved cluster, in contrast with the still actively accreting A1731.

Conclusions. Analysis of the specific star formation rate reveals evidence of ongoing galaxy pre-processing along A1731's filamentlike structure. Furthermore, the decrease in the number of star-forming galaxies and AGN towards the cluster cores suggests that the cluster environment is accelerating the ageing process of the galaxies and blocking further accretion of the cold gas that fuels both star formation and black hole accretion activity.
\end{abstract}

Key words. infrared: galaxies - galaxies: star formation - galaxies: active - galaxies: clusters: individual: Abell 983 galaxies: clusters: individual: Abell 1731 - galaxies: evolution

\section{Introduction}

The current paradigm of structure formation predicts that the galaxy population in clusters is evolving as new members are accreted from the surrounding field region (Balogh et al. 1998; Vogelsberger et al. 2014). The dependence of a galaxy's evolution on the environment in which it is located is proven to be tight. The morphology-density relation (Dressler 1980) implies that environment affects the star formation history, colour, and structure of galaxies. As a result, the young and active galaxies can typically be found in the cluster outskirts, and the passive ones in the cluster core. The processes that concur with this fast ageing of galaxies are several. The ram pressure that is due to the intracluster medium (ICM; Gunn \& Gott 1972; Steinhauser et al. 2012) builds on the gas that is present in the galaxy as it travels through the cluster. The consequent compression of the galaxy gas leads to sudden enhancement of the star formation (SF) but it also favours gas removal at later stages. Galaxies can also suffer gas losses via gravitational disturbance. Larson et al. (1980) noticed that the hot gaseous halo of the galaxy is stripped

\footnotetext{
* The catalogue and the reduced images (FITS files) are only available at the CDS via anonymous ftp to cdsarc.u-strasbg.fr (130.79.128.5) or via http://cdsarc.u-strasbg.fr/viz-bin/qcat?]/A+A/588/A105
}

as it enters the cluster environment. This process, called galaxy strangulation, prohibits further accretion of gas on the galaxy. Furthermore, the high density of galaxies in the cluster environment promotes frequent gravitational encounters, that induce the so-called harassment process. The dynamical equilibrium of the gas is altered and its collapse is facilitated because of the perturbation of the gravitational potential. This leads to new bursts of SF and hence further ejection of a portion of the remaining gas that is due to stellar winds. The efficiency of gravitational encounters in triggering new SF episodes increases in dense environments with low velocity dispersion. These conditions are found in the filaments, along which the galaxies are funneled and accreted in the cluster (Balogh et al. 2000; Diaferio et al. 2001; Okamoto \& Nagashima 2003). For example, Fadda et al. (2008) and Biviano et al. (2011) find a higher fraction of star-forming galaxies in the filament of Abell 1763, which is double that of the cluster core and outskirts. Wolf et al. (2009), Biviano et al. (2011), De Lucia et al. (2012), Wetzel et al. (2013) showed that long-lasting encounters are more likely to trigger SF in filaments than abrupt processes such as mergers (see also Wijesinghe et al. 2012). Haines et al. (2015) find the specific star formation rate (sSFR) of massive galaxies in a sample of 30 cluster as being $\sim 30 \%$ lower than their counterparts in the field. A subsequent modelling allowed to constrain the quenching timescale in the 


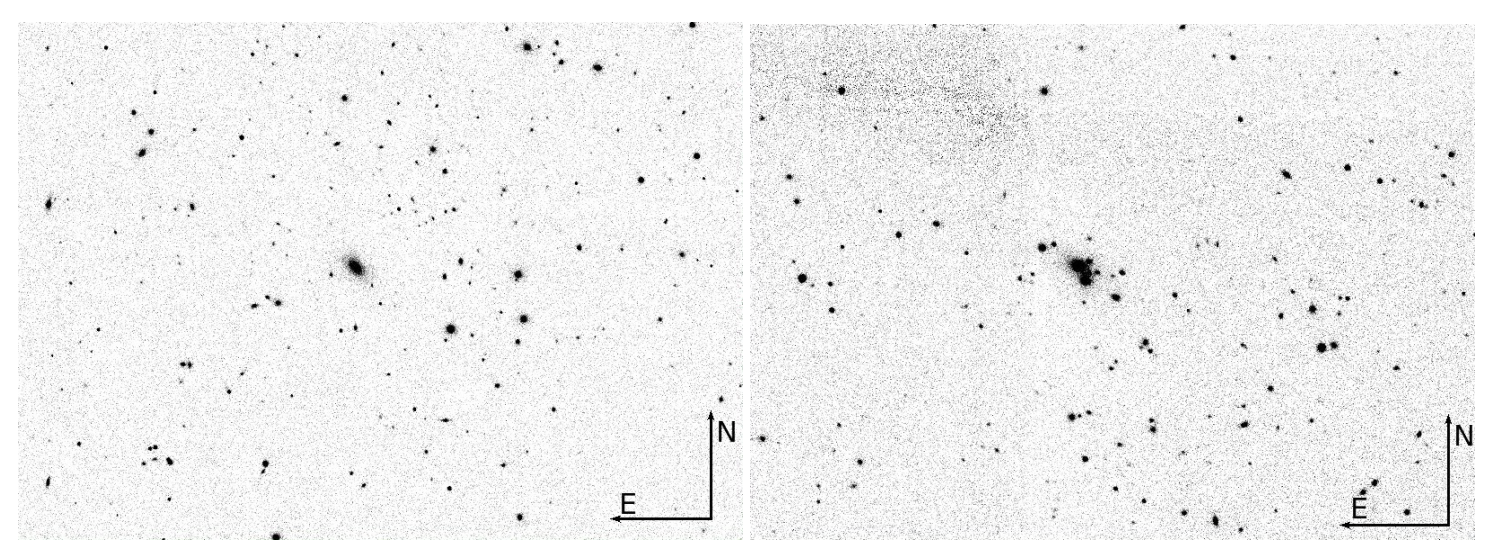

Fig. 1. WIRC Ks images of A983 (left panel) and A1731 (right panel), zoomed on the central $7^{\prime} \times 5^{\prime}$ region. The figures are centered on the brightest cluster galaxies.

Table 1. Main properties of the observed clusters.

\begin{tabular}{lccccc}
\hline \hline Cluster & $M_{200}\left[M_{\odot}\right]$ & $r_{200}[\mathrm{Mpc}]$ & $\sigma_{v}\left[\mathrm{~km} \mathrm{~s}^{-1}\right]$ & $\bar{z}$ & Members \\
\hline Abell 983 & $1.36 \times 10^{15}$ & 2.140 & 1071 & 0.20 & 134 \\
Abell 1731 & $1.92 \times 10^{15}$ & 2.408 & 1201 & 0.19 & 91 \\
\hline
\end{tabular}

range of 0.7-2.0 Gyr, consistent with the characteristic accretion timescale of galaxies in clusters. Peng et al. (2015) support the scenario in which local quiescent galaxies with stellar masses smaller than $10^{11} M_{\odot}$ (i.e. the vast majority of galaxies) are primarily quenched as a consequence of strangulation. To correctly interpret the evolution of galaxies and their accretion history, it is therefore fundamental to cover the entire extent of galaxy clusters observationally, with the inclusion of the outskirts and possible accretion structures. In addition, it is important to consider clusters that do not present evidence of ongoing mergers. The intense disturbance on cluster dynamics can hide or cancel the effects of the environment on galaxies and prohibit the study of the secular accretion of the galaxies. A fundamental difficulty in studying these objects is the contamination of interlopers, i.e. foreground and background galaxies. This issue becomes more important at larger clustercentric radii where the relative fraction of interlopers is larger. Therefore, spectroscopic redshift measurements are required.

The star formation rate (SFR) is an instantaneous quantity, directly susceptible to the influence of external processes, and therefore well suited for the study of environmental effects (Fadda \& Duc 2002). Robust measurements of the SFR are necessary, and hence must include both the obscured (via the infrared emission) and unobscured (via the UV and optical emission) star formation activity. In addition, recent studies correlate the presence and the characteristics of active galactic nuclei (AGN) with their parent galaxies, as well as to the environment in which these galaxies are located. So far, contradictory scenarios have been proposed in the literature, without any clear predominance (Sabater et al. 2013). The cluster environment enables us to study the duality of the environmental effects: on the large scale, influencing the gas supply, and on the local scale, regulating the accretion of the AGN via galaxygalaxy interactions.

The galaxy clusters Abell 983 and 1731 (hereafter A983 and A1731, respectively) are local rich galaxy clusters that we selected as targets for our study. ROSAT X-ray images of A983 reveal the uniform emission of the ICM, which traces the relaxed and virialised state of the cluster. On the other hand, A1731 shows signs of a less homogeneous X-ray surface brightness. However, the shallow depth of the X-ray data does not allow to draw firm conclusions on distribution of ICM. With respect to A983, A1731 presents a higher density of galaxies in the core, with two bright cluster galaxies that are surrounded by smaller objects (see Fig. 1).

In this paper, we present the study of star formation and black hole accretion activity in A983 and A1731. This study is based on deep infrared Spitzer observations, near-IR Palomar imaging and optical WIYN spectroscopy. This paper is structured in the following way. In Sect. 2, we present the observational program and the data reduction in detail. In Sect. 3, we present our matched photometric and spectroscopic catalogue. In Sect. 4, we present the results of the analysis of our dataset. In Sect. 5, we discuss the scenario that can be drawn from our results. In Sect. 6, we summarise the results and present future prospects for the project. Throughout this paper, we assume $H_{0}=70 \mathrm{~km} \mathrm{~s}^{-1} \mathrm{Mpc}^{-1}, \Omega_{\mathrm{M}}=0.3$ and $\Omega_{\Lambda}=0.7$. At the clusters' redshift, 1 arcsec corresponds to $\sim 3.2 \mathrm{kpc}$.

\section{Data set}

The main quantitative properties of A983 and A1731 are obtained in Sect. 5 and summarised in Table 1. The area covered by our photometric and spectroscopic observations is shown in Fig. 2. The main details of these observations are summarised in Tables 2 and 3. In the following sections, we introduce and describe the observing strategy and the subsequent data reduction and analysis that led to the production of the cluster members catalogues.

\subsection{Observations: mid- to far-IR with Spitzer IRAC and MIPS}

The galaxy clusters A983 and A1731 were observed as part of the Spitzer program 20512 (PI: Dario Fadda). A983 and A1731 were selected along with another cluster, Abell 1763 (Edwards et al. 2010), as being rich systems and located at a similar redshift in regions with extremely low Galactic emission, which allows us to measure the SF from the infrared with a lower 
M. Bianconi et al.: SF and $\mathrm{BH}$ accretion activity in rich local clusters of galaxies
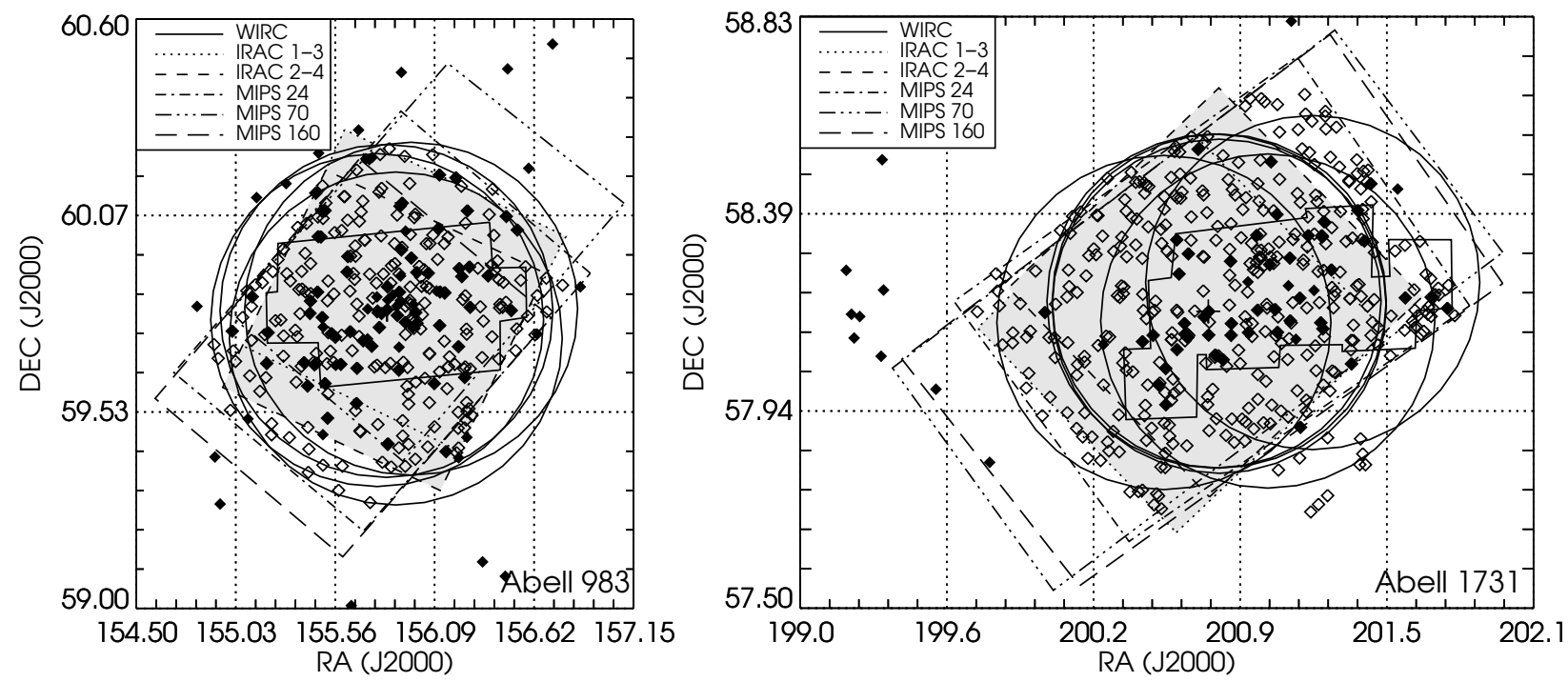

Fig. 2. Coverage of IR and optical data for A983 (left panel) and A1731 (right panel). The open and filled diamonds correspond to the HYDRA observed targets and the spectroscopically confirmed members, respectively. The solid line polygon delineates the WIRC observations. The shaded region marks the IRAC fields. The 3.6 and $5.8 \mu \mathrm{m}$ data are outlined with a dotted line, and the 4.5 and $8.0 \mu \mathrm{m}$ data are outlined with a dashed line. The footprints of the MIPS $24 \mu \mathrm{m}, 70 \mu \mathrm{m}$ and $160 \mu \mathrm{m}$ fields are delimited by the dot-dashed, dash-triple dot, and long dashed lines, respectively. The large circles indicates the WIYN/Hydra field of view.

Table 2. Near- to far-infrared observations of A983 and A1731.

\begin{tabular}{|c|c|c|c|c|c|c|}
\hline Cluster & Instrument & $\lambda_{\text {cent }}[\mu \mathrm{m}]$ & Date & Time $[\mathrm{min}]$ & Coverage $\left[{ }^{2}\right]$ & $F W H M$ of PSF ["] \\
\hline \multirow{7}{*}{ Abell 983} & IRAC & 3.64 .55 .88 .0 & 2005 Nov. 26 & 72.9 (each) & 1600 & 1.661 .721 .881 .98 \\
\hline & MIPS & 24 & 2006 May 07 & 498.6 & 2200 & 5.9 \\
\hline & MIPS & 70 & 2006 May 07 & 498.6 & 2035 & 16 \\
\hline & MIPS & 160 & 2006 May 07 & 498.6 & 2000 & 40 \\
\hline & WIRC $J$ & 1.250 & 2007 Mar. 26-27 & 61.0 & 1000 & 1.3 \\
\hline & WIRC $H$ & 1.635 & 2007 Mar. $26-27$ & 99.3 & 1000 & 1.2 \\
\hline & WIRC $K_{\mathrm{s}}$ & 2.150 & 2007 Mar. 26-27 & 67.2 & 1000 & 1.4 \\
\hline \multirow[t]{7}{*}{ Abell 1731} & IRAC & 3.64 .55 .88 .0 & 2005 Jun. 13 & 72.7 (each) & 1600 & 1.661 .721 .881 .98 \\
\hline & MIPS & 24 & 05 Dec. 08/06 Jun. 12 & 498.6 & 2200 & 5.9 \\
\hline & MIPS & 70 & 05 Dec. 08/06 Jun. 12 & 498.6 & 2880 & 16 \\
\hline & MIPS & 160 & 05 Dec. 08/06 Jun. 12 & 498.6 & 2700 & 40 \\
\hline & WIRC $J$ & 1.250 & 2008 Apr. 24-26 & 31.5 & 950 & 1.3 \\
\hline & WIRC $H$ & 1.635 & 2008 Apr. 24-26 & 31.5 & 950 & 1.2 \\
\hline & WIRC $K_{\mathrm{s}}$ & 2.150 & 2008 Apr. $24-26$ & 41.0 & 950 & 1.4 \\
\hline
\end{tabular}

limit of $\sim 1 M_{\odot} \mathrm{yr}^{-1}$. Furthermore, these clusters were targeted for being at low redshift $(z \sim 0.2)$, allowing a wide coverage that extends to approximately to three virial radii.

The Spitzer IRAC images were taken with the instrument set in mapping mode, allowing the simultaneous imaging of the field of view in the four different channels that correspond to $3.8,4.5,5.8$, and $8 \mu \mathrm{m}$. Each pointing of the telescope was dithered three times to allow for the detection and removal of transient phenomena, such as cosmic rays. The IRAC images cover $39.2 \times 39.2 \mathrm{sq}$. arcmin on the plane of the sky, corresponding to $7.3 \mathrm{Mpc} \times 7.3 \mathrm{sq}$. Mpc. The MIPS images were taken using slow telescope scanning, suitable for covering large portions of the sky. Throughout the scanning of the sky, the subsequent frames overlap each other, allowing a more efficient removal of cosmic rays. The motions of a secondary mirror in a cryogenic bath compensate for the oscillations of the telescope during the scanning. The MIPS images overlap the region covered by the IRAC images, and extend beyond that, reaching a size of $40.3 \times 55.6 \mathrm{sq}$. $\operatorname{arcmin}(7.5 \times 10.4 \mathrm{sq}$. Mpc $)$. In the case of A1731, the MIPS 70 and $160 \mu \mathrm{m}$ present a larger final footprint $\left(\sim 2880^{\prime 2}\right.$ and $\sim 2700^{\prime 2}$, respectively), owing to a shift of $\sim 18^{\prime}$ that occurred with the pointing of the last Astronomical Observational Request (AOR). In the $24 \mu \mathrm{m}$ band, we achieved a flux limit of $0.2 \mathrm{mJy}$ in $29.9 \mathrm{ks}$. Table 2 summarises the main properties of our near- to far-IR dataset. The full width at half maximum (FWHM) of the point spread function (PSF) for the Spitzer observations is quoted from the IRAC and MIPS Data Handbooks ${ }^{1}$.

The standard Spitzer pipeline processes the raw data, outputting basic calibrated datasets (BCDs). The corrections that were applied include dark subtraction, cosmic ray correction, detector linearization, flat field application and muxbleeding correction $^{2}$. The latter artifact consists of an electronic ghosting that can appear on the detector due to the delay of the detector in returning to its ground state, e.g. after the read-out of a bright source. We applied additional corrections to the BCDs, to obtain a higher signal-to-noise ratio $(\mathrm{S} / \mathrm{N})$ in the images and to avoid false source extraction when running automated software

\footnotetext{
1 http://irsa.ipac.caltech.edu/data/SPITZER/docs/ sitemap/

2 http://irsa.ipac.caltech.edu/data/SPITZER/docs/irac/ iracinstrumenthandbook/1
} 
Table 3. Details of the spectroscopic observations with HYDRA at WIYN.

\begin{tabular}{lcccc}
\hline \hline Cluster & Pointing & Date & FOV Centre [RA, Dec (J2000)] & Integration time [s] \\
\hline Abell 983 & 1 & 2006 Apr. 28 & $10: 23: 08.087+59: 46: 58.00$ & $3 \times 1200$ \\
& 2 & 2006 Apr. 29 & $10: 23: 31.913+59: 43: 58.00$ & $3 \times 1200$ \\
& 3 & 2006 Apr. 30 & $10: 23: 20.009+59: 48: 28.00$ & $3 \times 1200$ \\
Abell 1731 & 4 & 2008 Jan. 14 & $10: 23: 51.566+59: 48: 49.00$ & $2 \times 2200$ \\
& 1 & 2006 Apr. 29 & $13: 22: 09.007+58: 08: 32.16$ & $3 \times 1200$ \\
& 2 & 2006 Apr. 28 & $13: 23: 05.127+58: 11: 26.16$ & $3 \times 1200$ \\
& 3 & 2006 Apr. 28 & $13: 22: 59.819+58: 10: 44.16$ & $3 \times 1200$ \\
& 4 & 2006 Apr. 29 & $13: 23: 05.129+58: 10: 44.16$ & $3 \times 1200$ \\
& 5 & 2006 Apr. 30 & $13: 24: 39.957+58: 13: 56.16$ & $3 \times 1200$ \\
& 6 & 2006 Apr. 30 & $13: 23: 54.371+58: 08: 44.16$ & $3 \times 1200$ \\
\hline
\end{tabular}

for object detection. The additional corrections follow the procedure described in Fadda et al. (2006) and Edwards et al. (2010). For the IRAC bands, we corrected for column pulldown, jailbars, stray light and spurious effects of bright sources. We then applied a superflat to the IRAC Channel 3 and 4 BCDs. Specifically, each $\mathrm{BCD}$, after masking the bright sources, was divided by its median value. Then, the median value of all these BCDs was computed and each original BCD was divided by this superflat. This procedures helps in removing the background gradient that is present in the final mosaic. For the MIPS data, we corrected for jailbars, background discontinuities, and pixel distortions. We also applied a superflat and an additional flat accounting for the impurities on the cryogenic scan mirror. The MIPS astrometry was corrected by extracting the position of the sources in sets of 25 consecutive frames and matching them to the SDSS DR10 $r^{\prime}$-band catalogue (Ahn et al. 2014).

The IRAC sources were extracted using SExtractor (Bertin $\&$ Arnouts 1996). We measured aperture fluxes using a radius of 3 arcsec for each source with a S/N larger than 3.5 and multiplied the flux of each channel by the corresponding point source aperture correction (see Table 4), following Surace (2005). We computed aperture magnitudes using 9 arcsec radius for extended sources and we applied the extended sources aperture correction, following the instruction on the Spitzer's IRAC Handbook, for the galaxies presenting an evident extended structure ${ }^{3}(\sim 1 \%$ of the total number of sources).

The MIPS point sources were extracted using Starfinder (Diolaiti et al. 2000). This software allowed us to estimate the PSF directly from the image, accounting for the instrument design, using an iterative procedure. The flux was then measured using apertures with radii of 10, 16, and 20 arcsec for 24, 70, and $160 \mu \mathrm{m}$, respectively. Multiplicative aperture corrections and colour corrections were applied accordingly (see Table 4$)^{4}$. We checked for the presence of extended sources in the MIPS $24 \mu \mathrm{m}$ by PSF fitting and removing the point sources from the original image. We measured Petrosian fluxes of the extended sources ( $\sim 2 \%$ of the total number of sources) from the residual image using SExtractor.

\subsection{Observations: near-IR with Palomar/WIRC}

Measuring the near-IR emission is essential to estimate the stellar mass in galaxies. This is because most of the galaxy stellar mass is locked up in the evolved population that emits most of

\footnotetext{
3 http://irsa.ipac.caltech.edu/data/SPITZER/docs/irac/ iracinstrumenthandbook/30/

4 http://irsa.ipac.caltech.edu/data/SPITZER/docs/MIPS/ mipsinstrumenthandbook/50/
}

its light in the $K$-band (Kauffmann \& Charlot 1998). Therefore, deep observations in the near-IR are essential for obtaining a robust estimate of the galaxy stellar mass. We obtained near-IR images of the central $60^{\prime} \times 90^{\prime}$ region of Abell 983 and Abell 1731 using the Wide InfraRed Camera (WIRC) on the Palomar 200 inch telescope.

A983 was imaged during two nights of observations on 2007 March 26-27. The images of A1731 were obtained during a second run on 2008 April 24-26. The nights were photometric, with a seeing between 0.9 and $1.4^{\prime \prime}$ (see Table 2). Figure 1 shows the $K_{\mathrm{s}}$ images of the cluster central regions and Fig. 2 shows the footprints of the instrument. The observing strategy for the two runs was different. To observe A983, we scanned the field by moving the field of view along in three strips with fixed declination. The $J$ images were obtained with an exposure time of $40 \mathrm{~s}$. In $H$ and $K_{\mathrm{s}}$, two coadds of $30 \mathrm{~s}$ and four co-adds of $10 \mathrm{~s}$ each, respectively, were taken to avoid saturation. The total integration times for $J, H$, and $K_{\mathrm{s}}$ were 61,99 , and $67 \mathrm{~min}$, respectively. For the second run, A1731 was imaged using a 7-position dithering pattern centred on nine different subfields. In this case we used an exposure time of $30 \mathrm{~s}$ for $J$ and $H$ and three coadds of $13 \mathrm{~s}$ in the case of $K_{\mathrm{s}}$. Hence, the total integration time was $31.5 \mathrm{~min}$ for $J$ and $H$ and $41 \mathrm{~min}$ for $K_{\mathrm{s}}$. Dark frames were also obtained for each integration time.

The same data reduction technique was applied to all three bands using a pipeline developed and kindly provided by Tom Jarrett. A median dark frame was subtracted from the data frames, and correction terms for the flux nonlinearity were applied to correct the bias. A median sky made of a maximum of 10 frames was calculated and subtracted from the data frame before flat fielding. The astrometry was checked using a list of known stars from the Two Micron All Sky Survey (2MASS; Skrutskie et al. 2006) and corrected by accounting for the rotational offset of the telescope and compensating for the distortion of the WIRC instrument. SWarp (Bertin et al. 2002) was used to mosaic the frames together. Flux calibration was performed relative to the 2MASS catalog. The magnitude limit that we aimed to reach for these observations was $M^{*}+2$, where $M^{*}$ is the magnitude at the knee of the luminosity function (Edwards et al. 2010). For a cluster of galaxies at $z \sim 0.2$, a typical value is $K_{\mathrm{s}}^{*}=15.6$ (De Propris et al. 2003). The majority of the cluster galaxies, being passive, present typical near-IR colour indices of $J-K_{\mathrm{s}}=1.7$ and $H-K_{\mathrm{s}}=0.8$ (Fukugita et al. 1995). Hence, our near-IR photometry reaches (at $3 \sigma$ ) a depth of $J \sim 19.5$, $H \sim 18.5$, and $K_{\mathrm{s}} \sim 17.5$, extending approximately two magnitudes deeper than the 2MASS archival data (Fig. 3).

To compare our photometry with 2MASS, aperture magnitudes were extracted from the mosaicked $J, H$ and $K_{\mathrm{s}}$ images, using SExtractor and a 4 arcsec aperture radius. This aperture 
M. Bianconi et al.: SF and BH accretion activity in rich local clusters of galaxies
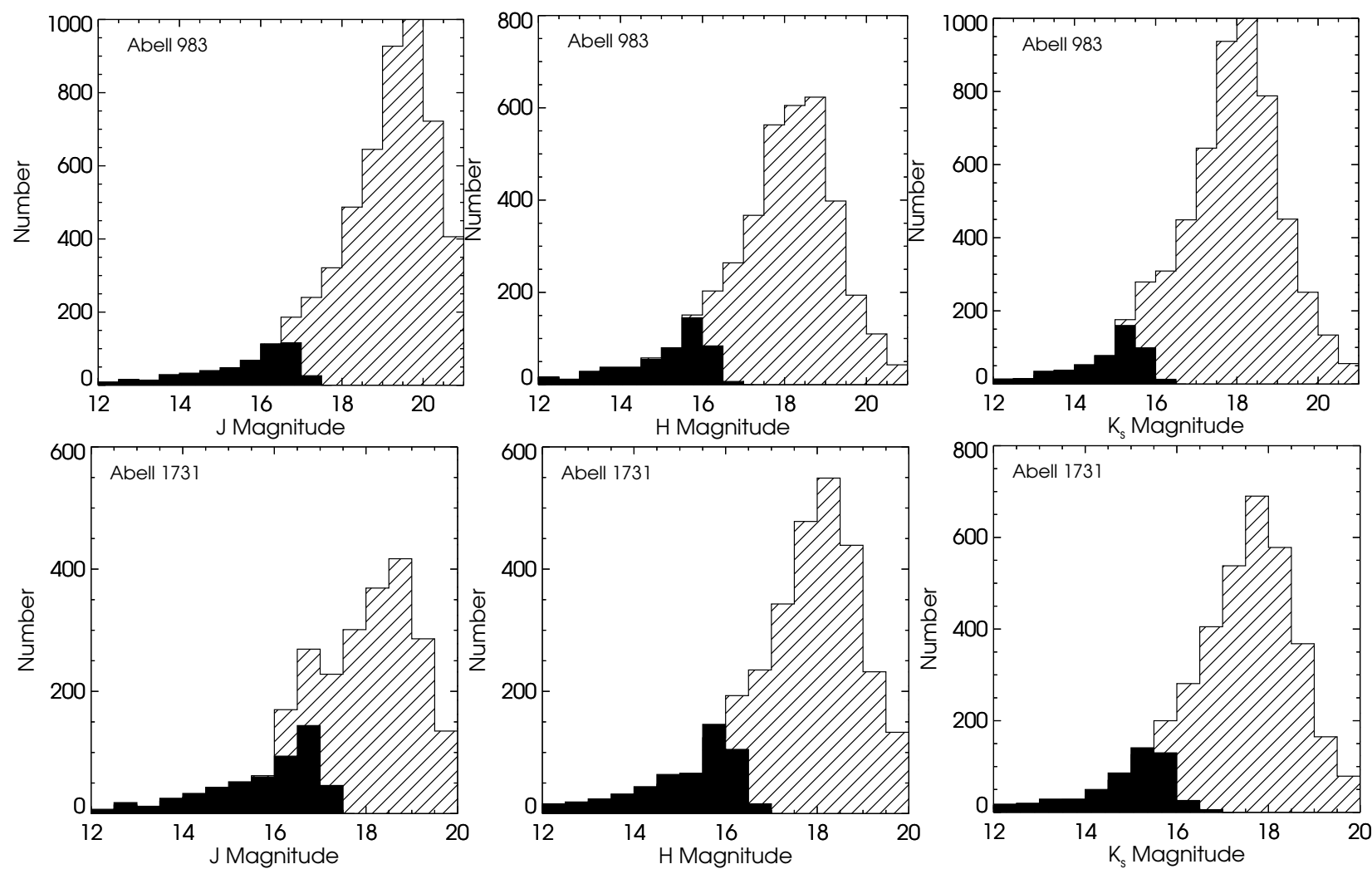

Fig. 3. WIRC $J, H, K_{\mathrm{s}}$ number counts for A983 (top) and A1731 (bottom). The histograms show the number of sources per bin of magnitudes $J$, $H$, and $K_{\mathrm{s}}$ from left to right, respectively. The overplotted filled histograms show the depth of the archival 2MASS data in the same region of the sky covered by the WIRC observations.

was chosen to match the one used in 2MASS. In Fig. 4, we present the comparison between our WIRC aperture magnitudes and the 2MASS ones. We measured a higher dispersion $(1 \sigma)$ of the difference between the two magnitudes at the faint end but limited to about $3.5 \%$ of the corresponding WIRC magnitude. This value decreases towards higher magnitudes $(<15)$ to about $2.5 \%$. The increasing scatter at the faint end is also due to the increase in the noise in the 2MASS data, which are close to the detection limit.

We then proceeded with the measurement of the Petrosian magnitudes from our mosaicked images using SExtractor. These magnitudes, used for the final catalogue, are measured on circular apertures within Petrosian radii. The Petrosian radius is defined as the radius at which the local surface brightness is a factor of 0.2 times the mean surface surface brightness inside the radius (Petrosian 1976). In order to be able to detect both faint and extended sources, we set a det_minarea of 5 and det_minthresh of 2.0. We used a deblend_mincont of 0.00005 to detect the smallest object that might be close to the largest galaxies.

\subsection{Observations: optical spectra at WIYN}

We obtained optical spectra of the MIPS $24 \mu \mathrm{m}$ sources with flux greater than $0.3 \mathrm{mJy}$ and optical magnitudes less than 20.5 in $r^{\prime}$ band in two different runs using the Hydra instrument mounted at the WIYN telescope at Kitt Peak National Observatory (KPNO). In total, we observed six and four overlapping pointings for A1731 and A983, respectively (see Fig. 2 and Table 3).

During the first run on 2006 April 28-30, we obtained spectra of all $24 \mu \mathrm{m}$ sources in A1731. We also observed three WIYN
Table 4. Aperture and colour corrections applied to the IRAC and MIPS sources.

\begin{tabular}{lccc}
\hline \hline Channel & $\begin{array}{c}\text { Aperture } \\
\text { radius ["] }\end{array}$ & $\begin{array}{c}\text { Aperture } \\
\text { corr. }\end{array}$ & $\begin{array}{c}\text { Colour } \\
\text { corr. }\end{array}$ \\
\hline IRAC 1 & 3 & 1.14 & $\ldots$ \\
IRAC 2 & 3 & 1.14 & $\ldots$ \\
IRAC 3 & 3 & 1.25 & $\ldots$ \\
IRAC 4 & 3 & 1.42 & $\ldots$ \\
MIPS 24 & 10 & 1.167 & 1.041 \\
MIPS 70 & 16 & 2.044 & 1.089 \\
MIPS 160 & 20 & 3.124 & 1.043 \\
\hline
\end{tabular}

fields of A983 targeting $8 \mu \mathrm{m}$ sources, since the MIPS observations were not yet available at the time of observation. During the second run on 2008 Jan. 14 we completed the observations of the $24 \mu \mathrm{m}$ sources in A983, which had not already been observed as $8 \mu \mathrm{m}$ emitters by observing a further 69 sources. To obtain spectra between 4000 and $9000 \AA$ we used the red cable and grating $316 @ 7.0$ at an angle of 21 degrees, centered at $6500 \AA$, and integrated for $3 \times 1200 \mathrm{~s}$. This gives a dispersion of $2.6 \AA$ pixel $^{-1}$, a spectral coverage of $5400 \AA$, and a resolution of $5.7 \AA$. The package DOHYDRA ${ }^{5}$ was used for the extraction and reduction of the spectra including the wavelength calibration, application of the flat-field and fibre throughput correction, and the sky subtraction. Cosmic rays were removed using the la_cosmic task (van Dokkum 2001). The scripts were run for each configuration and for each night. More details on the reduction methods

F. Valdes 1995, Guide to DOHYDRA, available at http://iraf. noao.edu/tutorials/dohydra/dohydra.html 

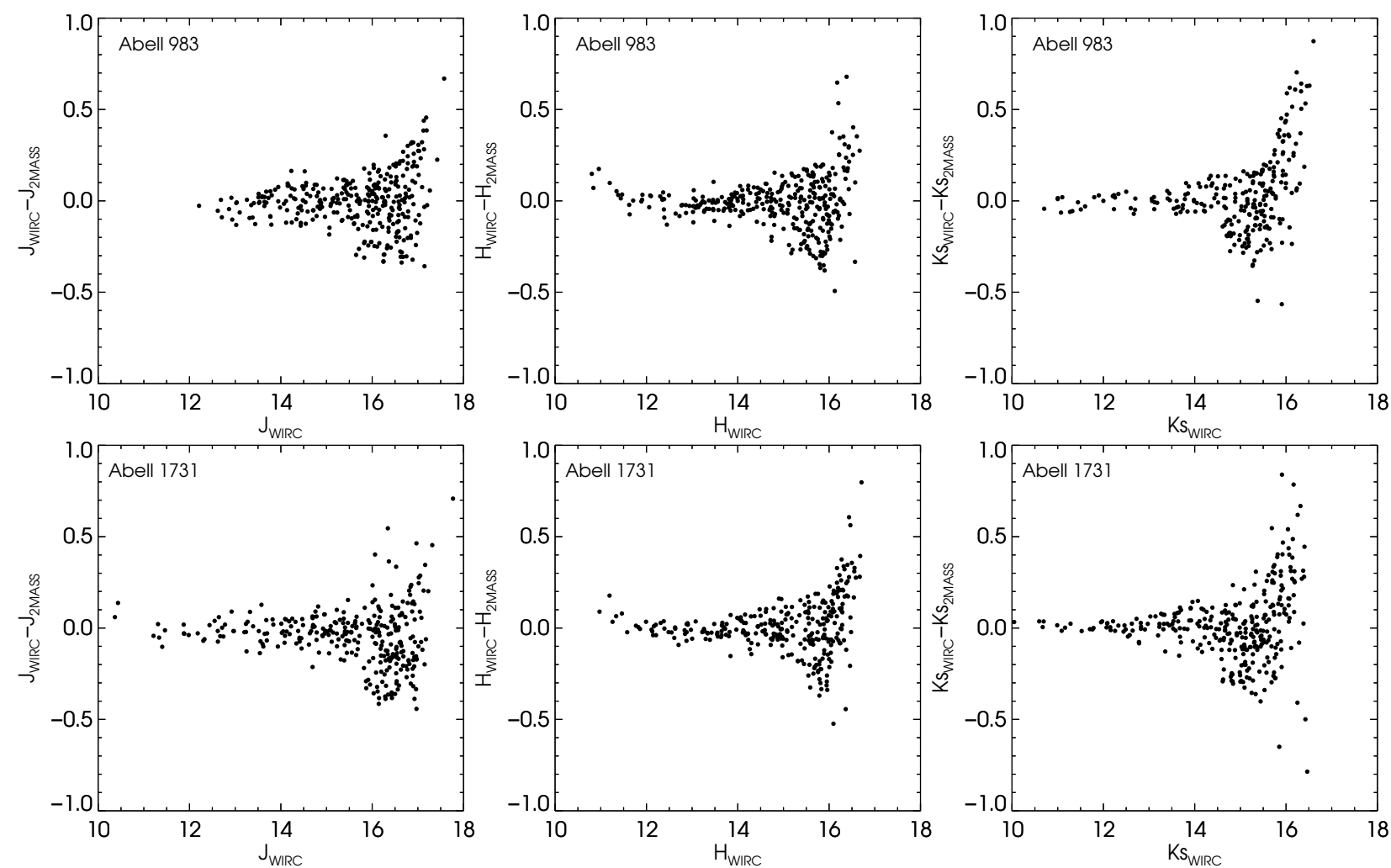

Fig. 4. Difference between the WIRC aperture magnitudes with the 2MASS aperture magnitudes, plotted against the IRC aperture magnitudes, for A983 (top) and A1731 (bottom). Each panel presents the sources in our field of view with a 2MASS counterpart and after the removal of close pairs.

can be found in Marleau et al. (2007). Taking the 101 available fibres into consideration, on average ten fibres were not usable and ten others were assigned to sky observation at each pointing. The number of $24 \mu \mathrm{m}$ target sources per configuration was less than the number of available fibres. Therefore, these free fibres were positioned on $r^{\prime}$-band sources with no $24 \mu \mathrm{m}$ counterpart (on average eight per configuration). We were able to obtain a total of 281 and 406 spectra for A983 and 1731, respectively. Of these, only ten and 25 spectra, respectively, did not have identifiable lines or continuum features.

We added archival SDSS DR10 spectra to our WIYN data. We selected the SDSS sources that are located in the cluster regions and extending up to $\sim 8 \mathrm{Mpc}\left(\sim 4 r_{200}\right)$ in clustercentric distance. Our aim was to increase the number of sources with spectroscopic data at the outskirts of the clusters. The line fitting was performed with an IDL code for both the WIYN and SDSS spectra. The code includes the Markwardt package algorithm for curve fitting. The region of the local continuum is selected by hand and fit with a straight line. The line is fitted on top of the continuum with a Gaussian function. In the case of blended lines, the code handles the fit of multiple Gaussian functions. The measured flux corresponds to the set of Gaussians that minimises the $\chi^{2}$ value of the fitted lines. The redshift of each line is allowed to vary and the final redshift assigned to the spectrum is the mean of the redshift of each line. The absorption features are included in the spectral templates. Owing to the level of noise, the fit to the absorption features was possible for only $4 \%$ of the total sample of spectra. Accounting for the absorption features helps in recovering the total line flux that is, otherwise, underestimated. This effect is more relevant at the high order of the Balmer series $(\mathrm{H} \delta, \mathrm{H} \gamma)$, where the fraction of the absorbed flux to the emission line flux is higher. The $\mathrm{H} \alpha$ and $\mathrm{H} \beta$ lines are dominated by the emission and the absorption accounts for $1 \%$ of the flux. In case of the $\mathrm{H} \alpha$ line, this translates to an average SFR of $0.05 M_{\odot} \mathrm{yr}^{-1}$. The emission line fluxes are used to estimate the star formation rate and their ratio is a stringent diagnostics of the presence of AGN. The average flux density limit that we achieved was $10^{-17} \mathrm{erg} \mathrm{cm}^{-2} \mathrm{~s}^{-1} \mathrm{~Hz}^{-1}$.

We compared our line fluxes to the SDSS photometry. For each WIYN fibre configuration, we measured the $r^{\prime}$-band magnitudes using the SDSS DR10 filter response function. A two arcsec aperture was used, corresponding to the diameter of the SDSS fibre. These factors were computed for each spectrum with an SDSS counterpart. The median value of this flux correction factor was then calculated and the flux of each spectrum, including those without an SDSS counterpart, was corrected accordingly. This correction factor ranged from 0.78 to 1.22 for both clusters. In addition, we applied an aperture correction to each $\mathrm{H} \alpha$ flux measurement. This aperture correction was obtained by taking the ratio of the fluxes extracted from the SDSS $r^{\prime}$-band image using a 2 and a 10 arcsec diameter aperture. The latter aperture was chosen since it corresponds to the average size of our sources.

\subsection{Archival data photometry}

We obtained archival data to complement the wavelength coverage of our observations. We retrieved the five optical bands $u^{\prime} g^{\prime} r^{\prime} i^{\prime} z^{\prime}$ from SDSS DR10, which are needed to measure a stellar mass estimate for each galaxy, using spectral energy distribution (SED) fitting. We also included the near-UV (NUV) band from GALEX, which contains the emission from newly formed stars. Additionally, we retrieved the archival WISE 3.6 and $4.6 \mu \mathrm{m}$ data. These bands were used to check the photometry 
of the IRAC 1 and 2 channels. The $1 \sigma$ dispersion of the difference, with respect to the IRAC magnitude, between the IRAC and WISE photometry ranged from $5 \%$ to $3.5 \%$ from low to high magnitudes, respectively.

\section{Cross-matching of the source catalogues}

We produced a final photometric catalogue using the positions of the MIPS $24 \mu \mathrm{m}$ sources as a reference with a $\mathrm{S} / \mathrm{N}$ in the first Airy ring ( $\sim 6$ arcsec) larger than 3 . The matching distance used corresponds to half of the first Airy ring ( $3 \mathrm{arcsec})$. The matching algorithm associates the position of the $24 \mu \mathrm{m}$ sources to sources detected in the NUV, the five SDSS optical bands, the three near-IR bands, the four mid-IR bands, the other two far-IR bands and the spectroscopic redshift. The spatial distribution of the sources is sparse in the field. Nonetheless, the FWHM of the MIPS $24 \mu \mathrm{m}$ is sufficiently large to hide close pairs of sources. A comparison with the IRAC images, which have a higher resolution, revealed that this problem affected only a small fraction of the total number of sources $(\sim 1 \%)$. The spectroscopically confirmed members (see Sect. 4.1) of the two clusters were not affected by this issue, since all of them have a unique counterpart in all the wavebands. Table 5 summarises the entries of our IR-selected source catalogue.

\section{Data analysis}

\subsection{The cluster membership}

The peculiar velocity of each galaxy was computed with respect to the mean cluster velocity $\bar{v}=\bar{z} c$, where $\bar{z}$ is the redshift of the cluster and $c$ is the speed of light. The redshift of the cluster was computed as the mean value of the redshifts in the range $0.19<z<0.21$. We used the shifting gap algorithm, as described in Fadda et al. (1996) to determine the cluster membership of the galaxies we observed. The shifting gap method makes use of both galaxy velocities and clustercentric distances. The clustercentric distance to each cluster galaxy was measured from the brightest cluster galaxy (BCG). The galaxies were grouped in overlapping and shifting bins of $500 \mathrm{kpc}$ from the cluster centre (or wide enough to contain at least 15 or 20 galaxies each for A983 and 1731, respectively). Then, gaps of $1000 \mathrm{~km} \mathrm{~s}^{-1}$ and $800 \mathrm{~km} \mathrm{~s}^{-1}$ for A983 and 1731 respectively, were searched in the observed galaxy velocity distribution. These gaps mark the separation between the velocity distribution of the cluster members and the external galaxies. This procedure rejects galaxies (interlopers) that have velocities bigger than $1000 \mathrm{~km} \mathrm{~s}^{-1}$ and $800 \mathrm{~km} \mathrm{~s}^{-1}$, for A983 and A1731, respectively. The procedure was reiterated until the number of cluster members converged. The advantage of this statistical method is the independence from any physical assumption on the dynamical state of cluster. The shifting gap method gives us a total of 134 and 91 members for A983 and 1731, respectively (see Fig. 5). The procedure was run iteratively until convergence on the number of selected members. By way of comparison, we also run the algorithm of Mamon et al. (2010). This code evaluates cluster membership from the distance-velocity space, based on models of mass and velocity anisotropy of cluster halos that are obtained from a cosmological simulation. This method produced 105 members and 77 members for A983 and 1731. The code outputs estimates for the virial mass, the velocity dispersion and $r_{200}$, and are quoted in Table 1. The bias towards late-type galaxies of our sample could lead to an overestimate of the velocity dispersion, as these
Table 5. The IR source catalogue columns.

\begin{tabular}{|c|c|c|}
\hline Column & Format & Description \\
\hline 1 & i6 & Catalogue Number \\
\hline 2 & a12 & RA (J2000) \\
\hline 3 & a12 & Dec. (J2000) \\
\hline 4 & $\mathrm{f} 10.3$ & MIP S24 ap $10^{\prime \prime}(\mu \mathrm{Jy})$ \\
\hline 5 & f10.3 & MIP S24 ap $10^{\prime \prime}$ error $(\mu \mathrm{Jy})$ \\
\hline 6 & f10.2 & GALEX NUV $(\mu \mathrm{Jy})$ \\
\hline 7 & f10.2 & GALEX NUV error $(\mu \mathrm{Jy})$ \\
\hline 8 & f10.3 & $u^{\prime}(\mu \mathrm{J} y)$ \\
\hline 9 & f10.3 & $u^{\prime}$ error $(\mu \mathrm{Jy})$ \\
\hline 10 & f10.3 & $g^{\prime}(\mu \mathrm{Jy})$ \\
\hline 11 & f10.3 & $g^{\prime}$ error $(\mu \mathrm{Jy})$ \\
\hline 11 & f10.3 & $r^{\prime}(\mu \mathrm{Jy})$ \\
\hline 13 & f10.3 & $r^{\prime}$ error $(\mu \mathrm{Jy})$ \\
\hline 14 & f10.3 & $i^{\prime}(\mu \mathrm{Jy})$ \\
\hline 15 & f10.3 & $i^{\prime}$ error $(\mu \mathrm{Jy})$ \\
\hline 16 & f10.3 & $z^{\prime}(\mu \mathrm{Jy})$ \\
\hline 17 & f10.3 & $z^{\prime}$ error $(\mu \mathrm{J} y)$ \\
\hline 18 & f10.3 & $J$ Petrosian $(\mu \mathrm{Jy})$ \\
\hline 19 & f10.3 & $J$ Petrosian error $(\mu \mathrm{Jy})$ \\
\hline 20 & f10.3 & $H$ Petrosian $(\mu \mathrm{Jy})$ \\
\hline 21 & f10.3 & $H$ Petrosian error $(\mu \mathrm{Jy})$ \\
\hline 22 & f10.3 & $K_{\mathrm{s}}$ Petrosian $(\mu \mathrm{Jy})$ \\
\hline 23 & f10.3 & $K_{\mathrm{s}}$ Petrosian error $(\mu \mathrm{Jy})$ \\
\hline 24 & $\mathrm{f} 10.2$ & IRAC 1 ap $3^{\prime \prime}(\mu \mathrm{Jy})$ \\
\hline 25 & $\mathrm{f} 10.2$ & IRAC 1 ap $3^{\prime \prime}$ error $(\mu \mathrm{Jy})$ \\
\hline 26 & f10.2 & WISE $1(\mu \mathrm{Jy})$ \\
\hline 27 & f10.2 & WISE 1 error $(\mu \mathrm{Jy})$ \\
\hline 28 & f10.2 & IRAC 2 ap $3^{\prime \prime}(\mu \mathrm{Jy})$ \\
\hline 29 & $\mathrm{f} 10.2$ & IRAC 2 ap $3^{\prime \prime}$ error $(\mu \mathrm{Jy})$ \\
\hline 30 & $\mathrm{f} 10.2$ & WISE $2(\mu \mathrm{Jy})$ \\
\hline 31 & $\mathrm{f} 10.2$ & WISE 2 error $(\mu \mathrm{Jy})$ \\
\hline 32 & $\mathrm{f} 10.2$ & IRAC 3 ap $3^{\prime \prime}(\mu \mathrm{Jy})$ \\
\hline 33 & $\mathrm{f} 10.2$ & IRAC 3 ap 3 " error $(\mu \mathrm{Jy})$ \\
\hline 34 & $\mathrm{f} 10.2$ & IRAC 4 ap $3^{\prime \prime}(\mu \mathrm{Jy})$ \\
\hline 35 & f10.2 & IRAC 4 ap $3^{\prime \prime}$ error $(\mu \mathrm{Jy})$ \\
\hline 36 & f10.2 & MIP S70 ap $16^{\prime \prime}(\mu \mathrm{Jy})$ \\
\hline 37 & f10.2 & MIP S70 ap $16^{\prime \prime}$ error $(\mu \mathrm{Jy})$ \\
\hline 38 & $\mathrm{f} 10.2$ & MIP S160 ap 20" $(\mu \mathrm{Jy})$ \\
\hline 39 & $\mathrm{f} 10.2$ & MIP S160 ap $20^{\prime \prime}$ error $(\mu \mathrm{Jy})$ \\
\hline 40 & f9.3 & MIP S24 S/N in 6" aperture \\
\hline
\end{tabular}

objects present more elongated orbits with respect to the passive population of galaxies (Biviano \& Katgert 2004). All members selected via the Mamon et al. (2010) method are included in the sample that was selected via the shifting gap algorithm. As the shifting gap method appears to be more conservative in identifying cluster members, specifically at high clustercentric distances, we keep this larger sample for our analysis.

\subsection{Stellar masses}

We estimated the stellar masses for each cluster member from the SED fitting, using the software MAGPHYS (da Cunha et al. 2008), with the addition of the stellar libraries by Bruzual \& Charlot (2003). The wavelength coverage (up to 16 bands) and the precision of the spectroscopic redshift ensure the high significance of the fit. As described in Sect. 2.3, the spectroscopic observations also targeted non-IR emitters. For these galaxies, we computed a separate SED fit that included only the bands from NUV to $K_{\mathrm{s}}$. 

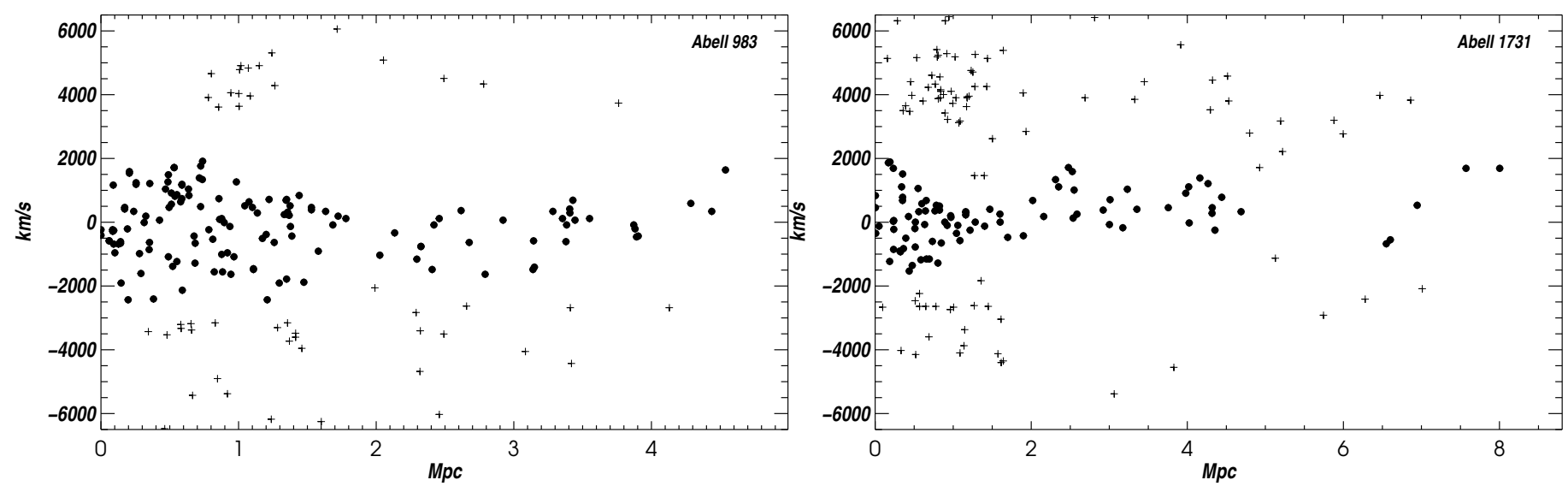

Fig. 5. Observed galaxies with a spectroscopic redshift in the range $0.15<z<0.25$, plotted in the clustercentric distance-velocity space. The filled dots correspond to the cluster members selected with the shifting gap method by Fadda et al. (1996). The right and left panels correspond to A983 and 1731 , respectively.

\subsection{AGN and star-forming galaxy separation}

The SFR values were retrieved using two different methods, i.e. using the computed luminosity in the infrared bands and the $\mathrm{H}_{\alpha}$ emission line flux. This two methods assume that the emission is dominated by star formation. Hence, before we applied these methods, we identified those objects whose emission was dominated by an AGN. We used three independent diagnostics for the detection of the AGN: the first is based on the characteristic ratio of optical emission lines, the second on identifying broad line AGN and the third utilises an infrared colour diagnostic.

\subsubsection{Emission line diagnostic}

The ratio of the fluxes of specific emission lines is useful for discerning the source of the ionizing radiation causing such lines. A clear signature of an active galactic nucleus is a high value for the flux ratio of $[\mathrm{NII}] / \mathrm{H} \alpha$ and $[\mathrm{OIII}] / \mathrm{H} \beta$ (Baldwin et al. 1981 ), with respect to more moderate values in the case of a star-forming region. To identify narrow-line AGN in our spectroscopic sample, we used the diagnostic diagrams of [NII]/H $\alpha$ versus $[\mathrm{OIII}] / \mathrm{H} \beta$ and $[\mathrm{OIII}] / \mathrm{H} \beta$ versus $[\mathrm{SII}] / \mathrm{H} \alpha$, applying the selection criterion of Kewley et al. (2001). These cuts result from the modelling of starburst galaxies with stellar population models (PEGASE version 2.0), producing the ionizing radiation, and with a detailed self-consistent photoionization model (MAPPINGS III). The AGN are modeled as $500 \mathrm{~km} \mathrm{~s}^{-1}$ radiative shocks (Kewley et al. 2001). For A983, we identified 11 and six AGN using the two diagrams with [NII] and [SII], respectively. Only two candidate AGN are common to both diagrams. In the case of A1731, we identified eight and seven AGN using both [NII] and [SII] diagrams, respectively (see Fig. 6). In this case, only one candidate AGN is common to both diagrams. We consider only the AGN selected via the [NII] as the [SII] lines (with observed wavelength $\sim 8000 \AA$ ) are located towards the end of the observed waveband range $(\sim 9000 \AA)$, where the sky emission lines dominate. Furthermore, Kewley et al. (2006) show that the [NII] selection method is more sensitive to low-energy AGN. In addition to these narrow line emission AGN, we visually identify five and four broad emission line AGN in A983 and 1731, respectively. Broad emission line AGN present permitted line widths in the range $10^{3}-10^{4} \mathrm{~km} \mathrm{~s}^{-1}$, while narrow
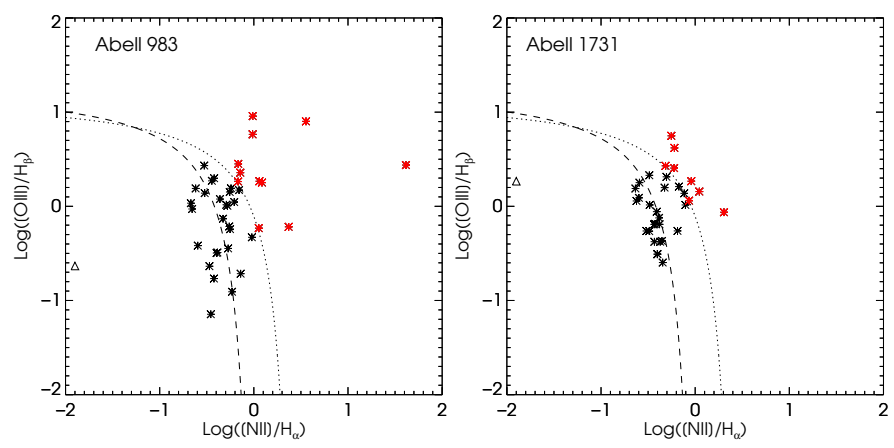

Fig. 6. Emission line diagnostic diagrams using $[\mathrm{NII}] / \mathrm{H}_{\alpha}$ and $[\mathrm{OIII}] / \mathrm{H}_{\beta}$ line ratios for $\mathrm{A} 983$ and $\mathrm{A} 1731$ in the left and right panel, respectively. The dotted line separates the HII star-forming regions, below, from the AGN, above, following the modelling by Kewley et al. (2001). The selected AGN candidates are plotted as red asterisks. The triangle identifies the source without and [NII] emission lines. As a comparison, we also plotted the Kauffmann et al. (2003) cut as dashed line, that selects less fiercely star-forming galaxies.

emission line AGN show permitted and forbidden line widths of $10^{2}-5 \times 10^{2} \mathrm{~km} \mathrm{~s}^{-1}$ (Hao et al. 2005).

\subsubsection{IR color diagnostic}

We used the IR colour selection proposed by Stern et al. (2005) to identify obscured AGN in our sample. The sources within the so-called Stern wedge are tagged as AGN. For each cluster, we find only one AGN candidate (see Fig. 7), which is not identified using the emission line diagnostic. In total we identify 17 AGN candidate in A983 and 13 in A1731.

\subsection{SFR from the total IR luminosity}

The use of MAGPHYS coupled with our multiwavelength catalogue (up to 16 bands from near-UV to mid-IR) ensures a consistent modelling of the galaxies, which includes the different phases of the ISM and the reprocessed star formation emission. We used the Kennicutt relationship (Kennicutt 1998) to translate the total infrared luminosity $L_{\mathrm{IR}}$ (from 8 to $1000 \mu \mathrm{m}$ ) estimated by MAGPHYS into a SFR:

$\operatorname{SFR}\left[M_{\odot} \mathrm{yr}^{-1}\right]=1.7 \times 10^{-10} L_{\mathrm{IR}} / L_{\odot}$ 

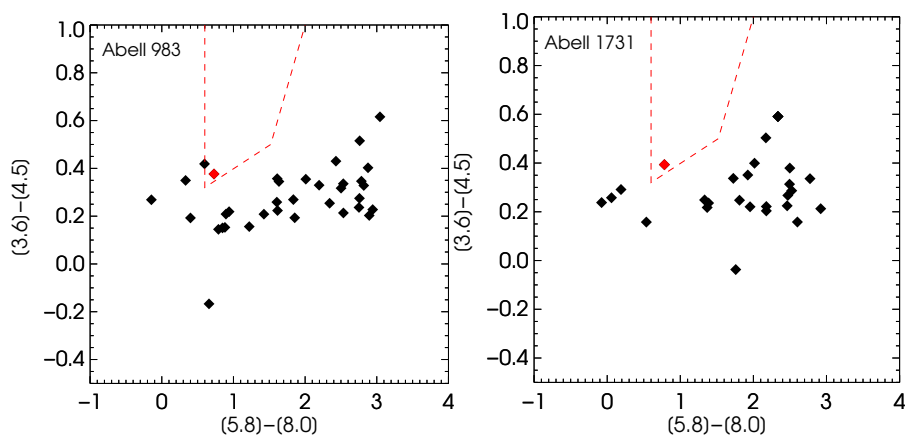

Fig. 7. AGN selected with the Stern et al. (2005) colour selection criteria. Each colour was evaluated by converting the flux in each of the IRAC channels into Vega magnitudes. Galaxies in A983 and 1731 are plotted in the left and right panels, respectively.

\subsection{SFR from the optical spectra}

\subsubsection{Extinction correction}

The emission line fluxes were corrected for the internal absorption of each galaxy due to the ISM. The extinction can be estimated using the Balmer decrement, i.e. comparing the observed and predicted Balmer line fluxes $(\mathrm{H} \alpha$ at $6563 \AA$ and $\mathrm{H} \beta$ at $4861 \AA$ ). A direct measurement of this decrement was possible for 53 and 66 galaxies out of 108 and 223 in the redshift range $0.15<z<0.25$ in A983 and A1731 field, respectively. We used the median value of the Balmer decrement of these subsets for the remaining galaxies in which one of the two lines was not detected. The colour excess $E(B-V)$ of each source is computed by comparing the ratio of the observed lines $\mathrm{H} \alpha$ and $\mathrm{H} \beta, F_{\mathrm{o}}^{\mathrm{H} \alpha, \beta}$, with the predicted unobscured value via the equation

$E(B-V)=\frac{2.5}{k(\mathrm{H} \alpha)-k(\mathrm{H} \beta)} \log \left(\frac{F_{\mathrm{o}}^{\mathrm{H} \alpha} / F_{\mathrm{o}}^{\mathrm{H} \beta}}{F_{i}^{\mathrm{H} \alpha} / F_{i}^{\mathrm{H} \beta}}\right)$

where $F_{i}^{\mathrm{H} \alpha, \beta}$ is the intrinsic unobscured flux of $\mathrm{H} \alpha$ and $\mathrm{H} \beta$, respectively, and $k(\lambda)$ is the reddening curve as a function of the wavelength. Here, the intrinsic unobscured line ratio $F_{i}^{\mathrm{H} \alpha} / F_{i}^{\mathrm{H} \beta}$ is set equal to 2.87, assuming case B recombination and $T=10^{4} \mathrm{~K}$ (Osterbrock 1989). The reddening curve $\mathrm{k}(\lambda)$ was taken from Calzetti et al. (2000) for starburst galaxies in the wavelength range from $0.12-2.2 \mu \mathrm{m}$. These quantities allow us to express the extinction as a function of wavelength via

$A(\lambda)=E(B-V) k(\lambda)$,

where $A(\lambda)$ is the mean extinction in units of magnitude at a specific wavelength $\lambda$. Using the IDL routine calz_unred, we computed the dereddened flux for each emission line. We assumed the default value for the effective total obscuration for starburst galaxies $R_{V}=4.05$, which include the effect of extinction, scattering, and the geometrical distribution of the dust relative to the emitters (Calzetti et al. 2000). The value of $A(\lambda)$ ranges between 0.5 and 3.5 and its mean is 1.5 .

\subsection{SFR from the $\mathrm{H}_{\alpha}$ emission line}

We calculated the SFR from the $\mathrm{H}_{\alpha}$ line flux (corrected for aperture and extinction) using the $\mathrm{H}_{\alpha}$-SFR relation derived by Kennicutt (1998):

$\operatorname{SFR}(\mathrm{H} \alpha)\left[M_{\odot} \mathrm{yr}^{-1}\right]=7.9 \times 10^{-42} L(\mathrm{H} \alpha)\left[\mathrm{erg} \mathrm{s}^{-1}\right]$
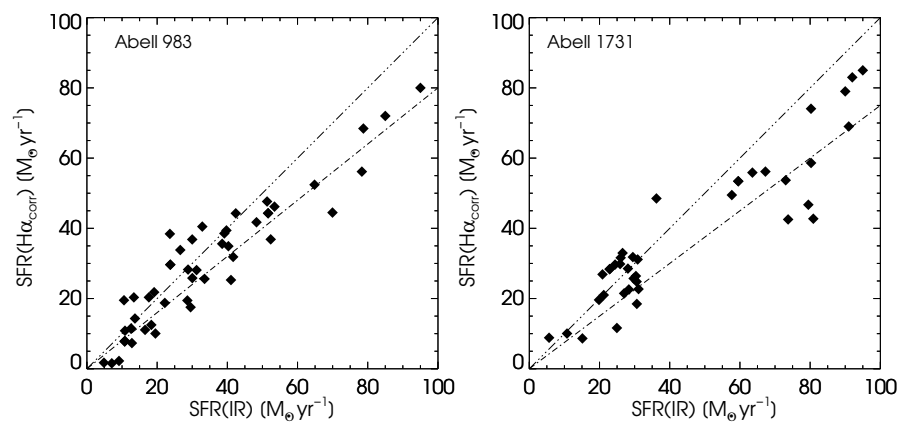

Fig. 8. Comparison of SFR evaluated from the extinction-corrected $\mathrm{H} \alpha$ line flux and the SFR based on the total IR luminosity obtained from the SED fit. The triple-dot-dash line corresponds to the relation with slope $m=1$. The dot-dashed line corresponds to the linear fit of the data. ( $m=0.8 \pm 0.02$ and $m=0.74 \pm 0.06$ for A983 and A1731, respectively). The left and right panels refer to A983 and 1731, respectively.

This relation applies especially when considering young and massive stellar populations, based on the assumption that the emission lines are tracers of the ionizing flux from newly formed stars. Equation (4) was introduced and calibrated using a Salpeter initial mass function (IMF) and over the mass range $0.1<M / M_{\odot}<100$. We estimated star formation rates in the range $0.1-200 M_{\odot} \mathrm{yr}^{-1}$. In agreement with Marleau et al. (2007), we find that these SFR are strongly correlated with extinction.

\section{Results}

\subsection{SFR from IR vs. $\mathrm{H} \alpha$}

Figure 8 shows the comparison of the SFR that was obtained from the IR luminosity and from the extinction corrected $\mathrm{H} \alpha$ line flux. An underestimate of the corrected flux, and hence of the SFR that was computed using the $\mathrm{H} \alpha$, can be seen for galaxies with $S F R>30 M_{\odot} \mathrm{yr}^{-1}$. As a consequence, the linear fit of the data presents a slope of $m=0.8 \pm 0.02$ and $m=0.74 \pm 0.06$ for A983 and A1731, respectively. The extinction correction is more effective for normal galaxies than for high star-forming dusty ones (Calzetti et al. 2000). To properly account for the obscured and unobscured star formation, we added the SFR from the total IR luminosity to the uncorrected $\mathrm{H}_{\alpha}$ emission, following Kennicutt et al. (2009). The limit of complete obscuration is satisfied in the most active galaxies, luminous and ultra-luminous infrared galaxies. For these objects, the SFR was evaluated using only the total IR luminosity. The SFR of the galaxies with only optical spectral lines was retrieved from the corrected $\mathrm{H} \alpha$ emission.

Figure 9 presents the $M_{*}-S F R$ relation for the spectroscopically-confirmed members, along with the $M_{*}$ and SFR distributions for each of the two clusters. To make a comparison, we overplotted the relation obtained by Noeske et al. (2007), who used a sample of field galaxies at redshift of $0.2<$ $z<0.45$. The different colours encode the methods used for estimating the SFR. For both clusters, the mean SFR of the members is compatible with the mean SFR of a coeval sample of field objects $\left(\log (S F R) \sim 0.8 M_{\odot} \mathrm{yr}^{-1}\right.$ for the clusters with respect to $\log (\mathrm{s} S F R) \sim 0.5 M_{\odot} \mathrm{yr}^{-1}$ for the field).

\subsection{Effect of the dynamical state of the cluster on SFR}

The clusters that we consider present a clear difference in their dynamical state. This difference is evident in the comparison of 

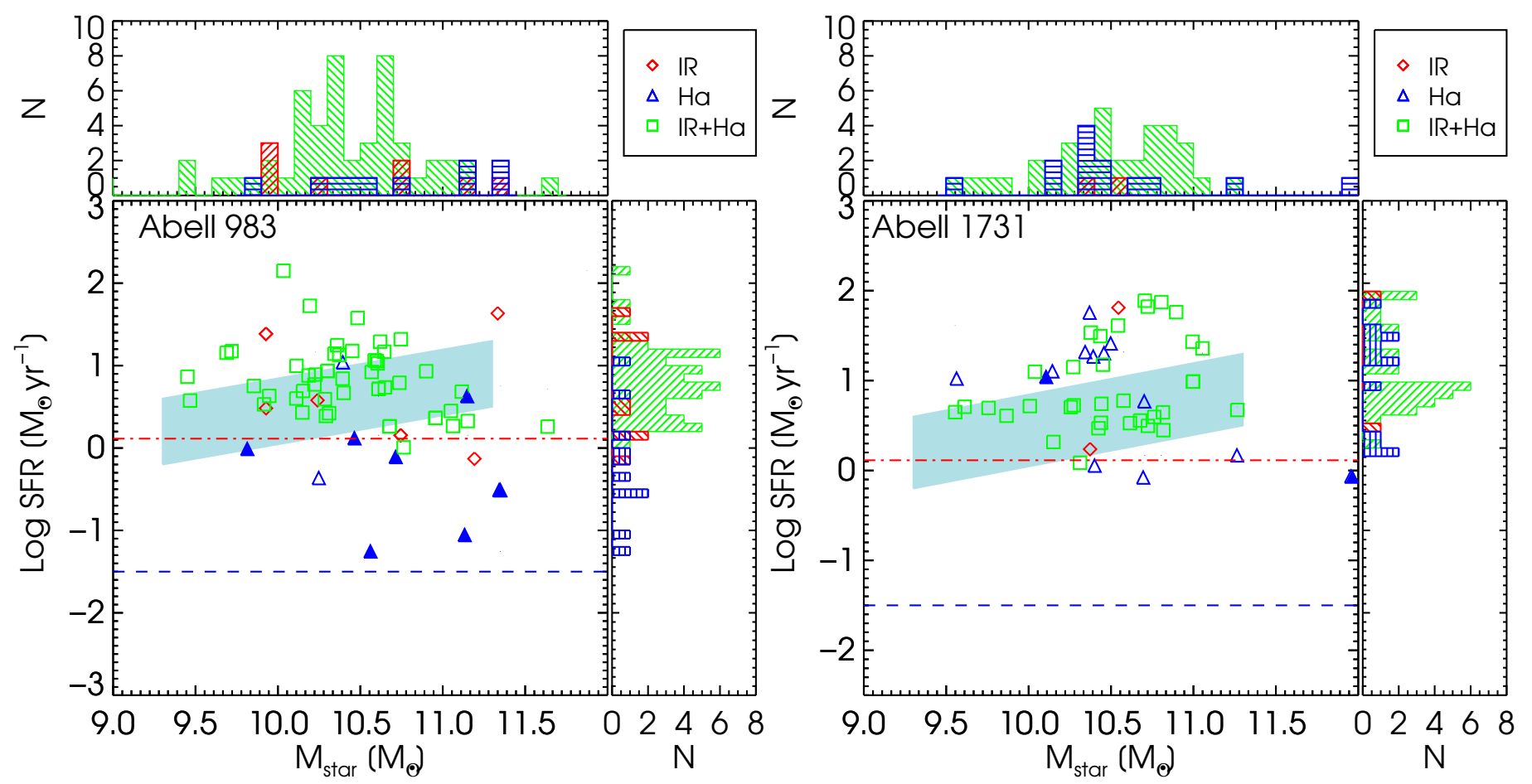

Fig. 9. $M_{*}-S F R$ relation for A983 and 1731 on the left and right panels, respectively. The histograms on the top and right side of each plot show the stellar mass and SFR distribution of the members. The SFR from only the total IR luminosity is plotted as red diamonds, the SFR from the extinction corrected $\mathrm{H} \alpha$ as open blue triangles, the combined SFR as open green squares. The filled blue triangles show the sources for which the mean extinction correction was used. The light blue shaded area corresponds to the relation with uncertainties found by Noeske et al. (2007). The red dot-dashed and the blue dashed lines indicate the SFR limit evaluated from the detection limit of the IR $24 \mu \mathrm{m}$ and optical spectral S/N, respectively.

the clustercentric-velocity plot (Fig. 5) of the two clusters. The cluster A983 presents the classical trumpet-like shape of galaxy distribution, with a clear separation between the hosted objects and the external ones. A1731 shows a less uniform distribution of its members. The central high number density of members becomes more sparse at a clustercentric distance of about $\sim 2 \mathrm{Mpc}$ $\sim 1 r_{200}$ ). The analysis of the peculiar velocity of A1731 galaxy members allows us to exclude the presence of neighbouring clusters or recent mergers, as no clear subclumps of galaxies are present. Haines et al. (2015) showed that the accreting objects are supposed to be found at these characteristic radii, comparing the cluster in the LoCuSS survey and the 75 more massive clusters in the Millennium simulation (Springel et al. 2005). This might suggest that A1731 is still actively accreting galaxies from the field.

Figure 10 presents the correlation between sSFR, defined as $S F R / M_{\star}$, and $M_{\star}$. The sSFR quantifies the instantaneous growth rate per stellar mass of a galaxy. We compare our results with the relation found by Oliver et al. (2010). They find a much flatter relation using a sample of galaxies $(0.2<z<0.3)$ from the Spitzer Wide-area InfraRed Extragalactic Legacy Survey. This difference can be explained by taking the different selection criteria of our work (24 $\mu$ m galaxies) and of Oliver et al. (2010) into consideration, which uses a $M_{*}$ selected sample. Therefore, our sample is incomplete at the low-mass end. The dot-dashed line in Fig. 10 represents the average expected relation between sSFR and $M_{*}$ for a $24 \mu \mathrm{m}$ source of $0.2 \mathrm{mJy}$ flux density, corresponding to the limit below which our sample becomes incomplete (see Biviano et al. 2011). This limit was computed using the relation of Lee et al. (2010) between the $f_{24}$ and $L_{\mathrm{IR}}$ at the average redshift of A983 and 1731, and the Kennicutt (1998) relation. For A983, the slope of the relation is close to the one found by Biviano et al. (2011). A1731 departs from this trend, presenting a higher fraction of galaxies with high sSFR. To quantify this difference, we selected galaxies in the mass range $10^{10}<M_{*}\left[M_{\odot}\right]<10^{11}$. We selected this interval since the low-mass end of our members sample could be incomplete, and the high mass end is affected by the intrinsic low number of objects. We performed a t-test to verify the significance of the higher mean value of the SSFR of A1731 $\left(\log (\mathrm{s} S F R)=0.1 \mathrm{Gyr}^{-1}\right)$ with respect to A983 $\left(\log (\mathrm{s} S F R)=-0.1 \mathrm{Gyr}^{-1}\right)$, finding a low value $(0.5)$ of significance. Therefore, the two distributions are not independent of one another. Interestingly, we found that $\sim 50 \%$ of the galaxies in this mass range are located at intermediate clustercentric distances $\sim 2-3$ Mpc $\left(\sim 1-1.5 r_{200}\right)$, for both clusters. This is compatible with the findings of interacting star-forming galaxies at the cluster outskirts by Haines et al. (2015). The newly accreted objects can also present on average higher sSFR values with respect to the cluster galaxies since they are not yet fully affected by the environment and hence have conserved the characteristics of field galaxies (Cohen et al. 2015). Figure 11 shows the 1D trend of the star-forming galaxies, selected as having $S F R \gtrsim 2 M_{\odot} \mathrm{yr}^{-1}$, with respect to the total number of members for each cluster. A983 presents a lower fraction of star-forming galaxies in the central region, which increases and reaches the maximum value of approximately $90 \%$ at $3-4 \mathrm{Mpc}$ $\left(\sim 1.5-2 r_{200}\right)$. Similarly, A1731 presents a central dip in the fraction of star-forming objects, but the overall distribution is flatter with respect to A983 and has a higher mean value ( $60 \%$ for A1731 and approximately 45\% for A983). We emphasise that the outskirts of the clusters suffer from the lower coverage and lower sensitivity of SDSS data, and might therefore be severely incomplete.

Figure 12 shows the 2D distribution of the sSFR. A clear asymmetry is present in the spatial distribution of the spectroscopic members of A1731, extending towards the north-west 
M. Bianconi et al.: SF and $\mathrm{BH}$ accretion activity in rich local clusters of galaxies
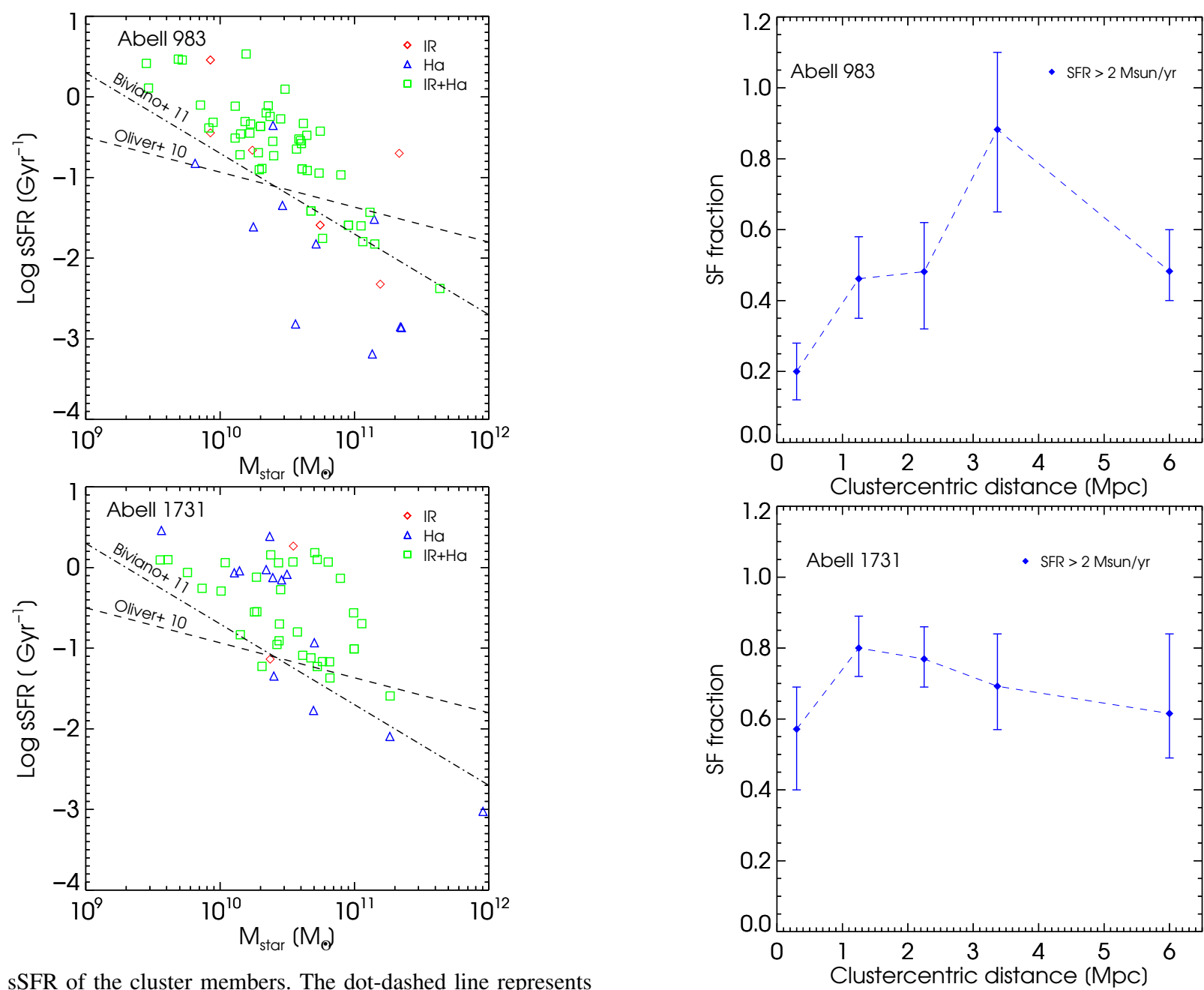

Fig. 10. sSFR of the cluster members. The dot-dashed line represents the expected sSFR vs. $M_{*}$ relation for a $24 \mu \mathrm{m}$ source of $0.2 \mathrm{mJy}$ flux density (i.e. at the completeness limit of our Spitzer observations), obtained in Biviano et al. (2011) using the relations of Lee et al. (2010) and Kennicutt (1998). The dashed line corresponds to the relation of Oliver et al. (2010) for galaxies from the Spitzer Wide-area InfraRed Extragalactic Legacy Survey in the redshift range $0.2<z<0.3$. The galaxies in A983 and 1731 are plotted in the top and bottom panels, respectively.

direction. Within this extended structure, $\sim 50 \%$ the star-forming galaxies present a value of the sSFR in the range $-10.5<$ $\mathrm{S} S F R\left[\mathrm{yr}^{-1}\right]<-9.3$, with respect to the remaining fraction that presents $\mathrm{S} S F R\left[\mathrm{yr}^{-1}\right]>-9.3$. Fadda et al. (2006) and Edwards et al. (2010) pointed out that accreted galaxies undergo an accelerated evolution when they meet the denser cluster environment, experiencing a short starburst phase followed by the quenching of their star formation activity. Cohen et al. (2014) and Cohen et al. (2015) find a higher fraction of star-forming galaxies in dynamically active clusters than in more relaxed ones, by measuring star formation with optical spectroscopic data from SDSS and infrared data from WISE. The lower SSFR in A1731 in half the galaxies at these intermediate clustercentric distances $\left(\sim 1.5-2 r_{200}\right)$ could be due to the ongoing harassment process, that leads to the consumption of the gas reservoir in the infalling galaxies and the reduction of the sSFR. This extended structure presents the spatial characteristic and the preprocessing evidence (Haines et al. 2015) of a filament-like structure. Additional spectroscopic observations and X-ray imaging

Fig. 11. Radially binned 1D fraction of star-forming galaxies $(S F R>$ $2 M_{\odot} \mathrm{yr}^{-1}$ ) with respect to the total number of galaxy members, plotted against the clustercentric distance. The errorbars are computed using the bootstrap method. The top and bottom panels refer to A983 and A1731, respectively.

would complement the currently available dataset, highlighting the presence of infalling groups of galaxies or a filament.

As an additional comparison, we built the colour-magnitude diagram of the $g^{\prime}-r^{\prime}$ versus $r^{\prime}$ magnitude from SDSS DR10 of the galaxies in the same region of the sky with respect to the clusters (Fig. 13). The passive population of the member galaxies falls into what is called a red sequence, which is clearly separated from the more star-forming and, therefore, bluer cloud of field galaxies. We visually selected the galaxies in the red sequence down to a $r^{\prime}=18$. We performed a linear fit of this subsample, and extended the relation to the whole sample, applying a $1 \sigma$ error cut above and below the fitted line. The selected passive population of galaxies is visible in Fig. 13. We compared the density distribution of the red-sequence galaxies to the distribution of the galaxies selected via the shifting gap method (see Fig. 14). Our aim is to verify the match between the evolutionary and dynamical centre of the clusters. A983 presents a direct match between the evolutionary and the dynamical centre (offset $\left.\sim 1^{\prime}\right)$. A1731 shows a similar offset $\left(\sim 2^{\prime}\right)$, although presenting less passive galaxies in the central $1 \mathrm{Mpc}$ region (270 and 120 galaxies in A983 and 1731, respectively). The extended structure, shown in Fig. 12, does not correspond to any overdense region of the passive galaxies. The scenario of 

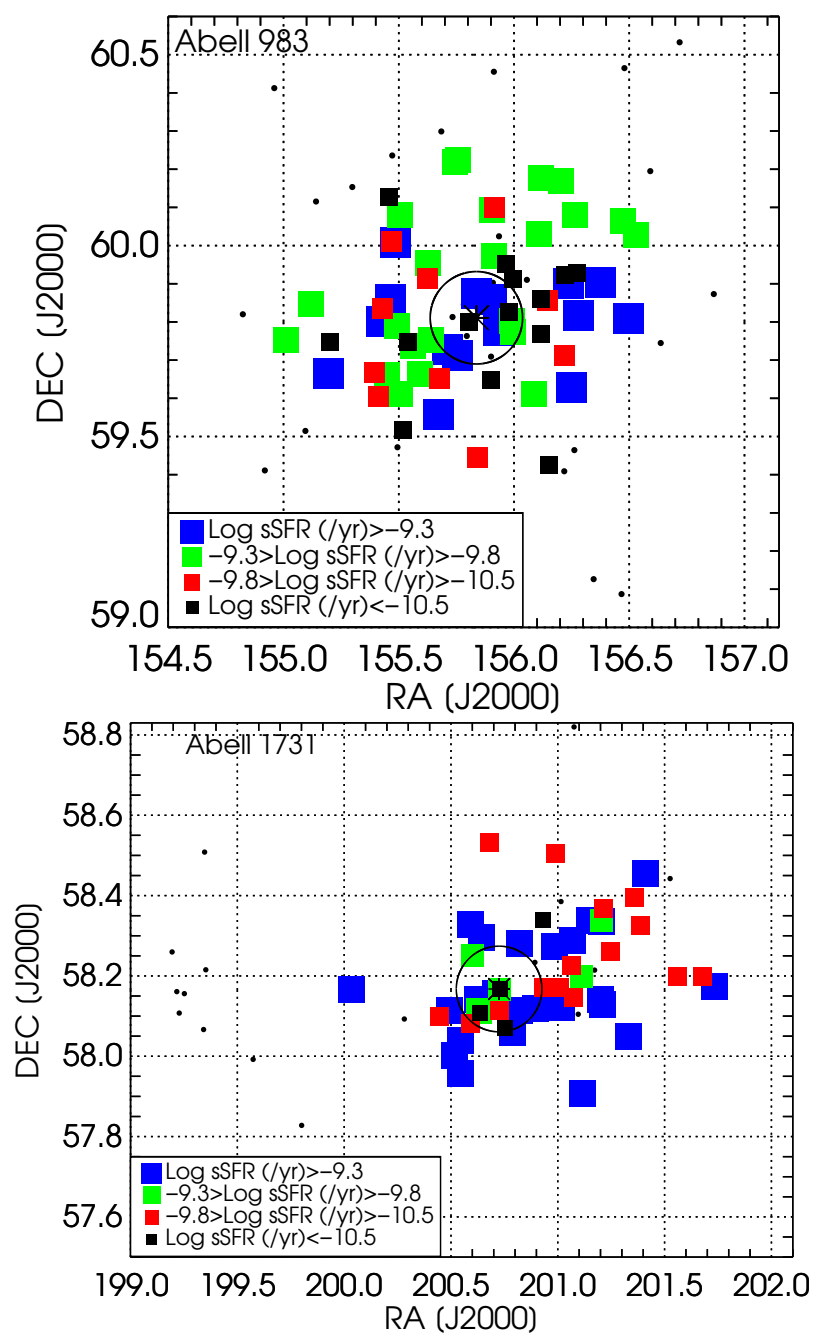

Fig. 12. 2D distribution of the sSFR of the members in the central region of A983 (top) and A1731 (bottom). The black dots indicate the members for which no star formation was measured. The BCG is marked as an asterisk and the open cirle represents $1.3 \mathrm{Mpc}$ distance from the cluster centre.

an actively accreting cluster therefore appears the most likely. The dense clump in Fig. 14, located at RA = 201.3 Dec. $=58.1$, presents no clear identification. The NED archive suggests the presence of a galaxy group in proximity to those coordinates (McConnachie et al. 2009). The overdensity at RA = 199.7 Dec. $=58.0$ corresponds to the cluster Abell 1713, located at $z \sim 0.14$.

\subsection{Effect of the dynamical state of the cluster on the AGN population}

Gravitational and hydrodynamical interactions are known to cause the disturbance of the gas content in galaxies. The compression and collapse of gas in the central region of a galaxy can trigger AGN activity (Springel et al. 2005; Hong et al. 2015) On the other hand, ram pressure stripping, although more efficient in depleting the outskirts of galaxies as they enter the cluster environment, could influence the inner reservoir of gas that powers the AGN. In Fig. 15, both clusters show a dearth of AGN in the cluster centre ( $1 \mathrm{Mpc}, \sim 0.5 r_{200}$, from the BCG), followed by an increase at the outskirts $\left(2-4 \mathrm{Mpc}, \sim 1-2 r_{200}\right.$, from the BCG).
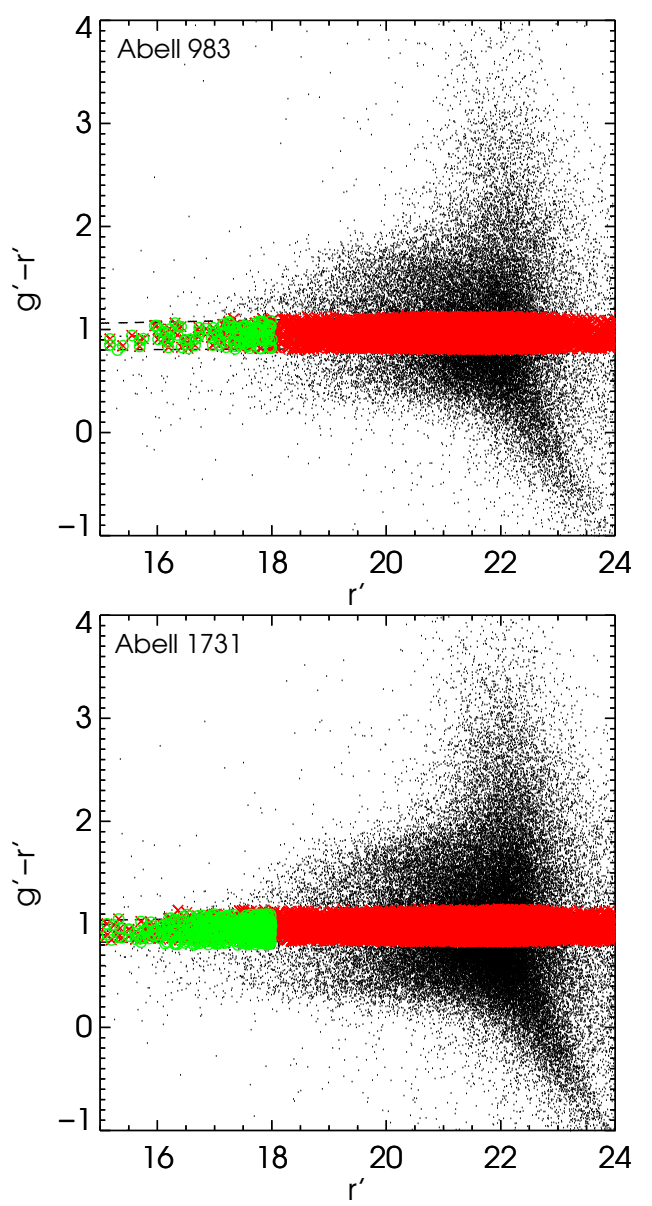

Fig. 13. Colour-magnitude diagram of the cluster A983 (top panel) and 1731 (bottom panel). The galaxies belonging to the red sequence are indicated with red crosses. The subsample of the galaxies used for the red-sequence selection is visible as green circles.

At higher clustercentric distances (larger than $5 \mathrm{Mpc}, \sim 2.5 r_{200}$, from the BCG), the clusters present a different behavior, with A983 showing a steep decrease in the AGN fraction, whereas A1731 shows a slight increase (from $10 \%$ to $16 \%$ ). Both clusters present no significant difference when comparing the cluster central and distant, field-like, regions, but a steep increase in the number of AGN is found on average at about $2 \mathrm{Mpc}\left(\sim 1 r_{200}\right)$ from the BCG. This result is in agreement with the results of Haines et al. (2013) and Pimbblet et al. (2013). At intermediate radii, the frequent gravitational interactions of gas-rich galaxies might favour the growth of instabilities in the gas distribution. These are accreted on the central black hole, causing an increase of its activity. Our study confirms that the majority of the active AGN are found at intermediate $\left(\sim 1-2 r_{200}\right)$ clustercentric distances, where the infalling galaxies still have to be fully processed in the cluster environment. This also holds when taking the non symmetrical distribution of the members in A1731 into consideration, indicating a relation between the AGN fueling efficiency and the global density of the ICM. The dual effect of the cluster environment on the AGN life cycle appears confirmed. On the local scale, the high density of galaxies promotes the instability and accretion of gas on the central black hole. On the large scale, the interaction with the ICM inhibits the subsequent replenishment of the gas reservoir. 

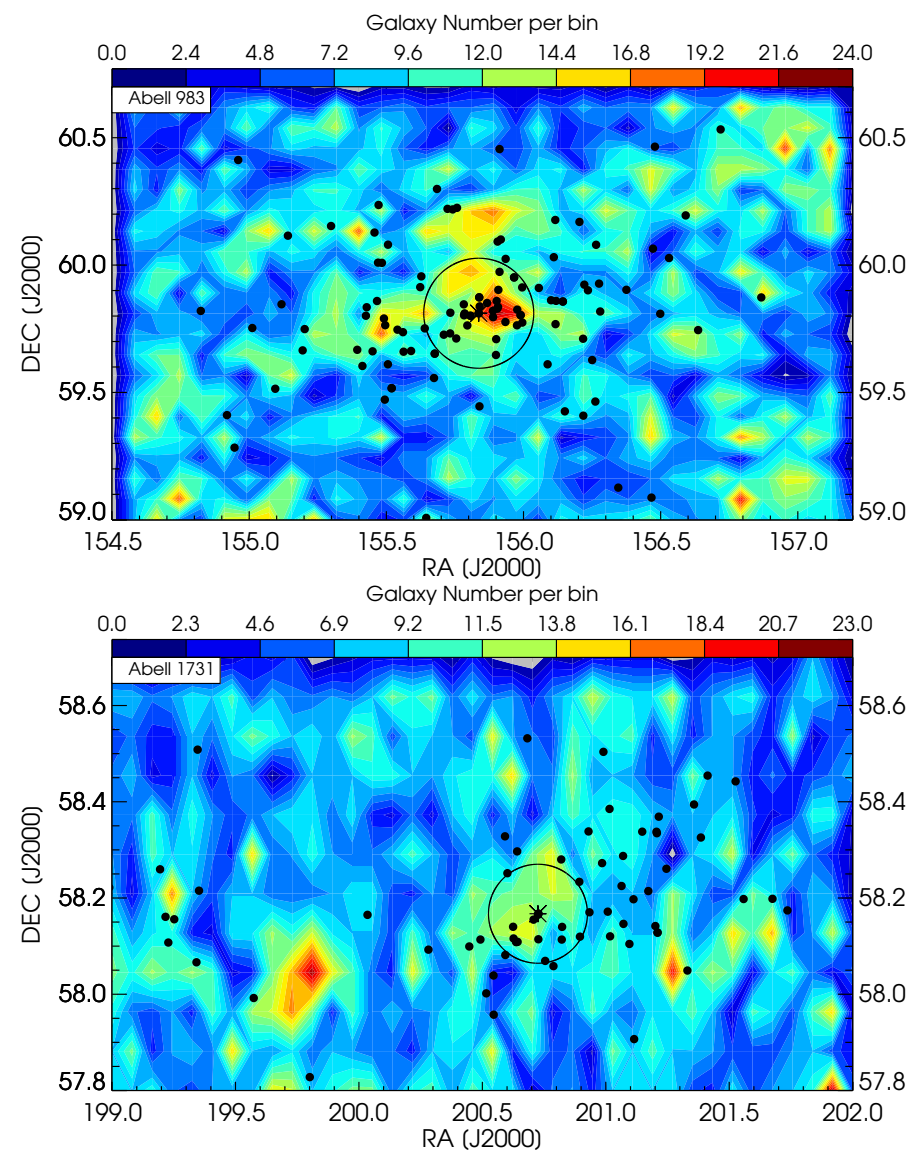

Fig. 14. 2D density distribution of the passive galaxies vs. the dynamical centre of the clusters (plotted as an asterisk). The spectroscopically confirmed members are overplotted as black circles. The panels for A983 and 1731 are the top and bottom one, respectively. The open cirle represents $1.3 \mathrm{Mpc}$ distance from the cluster center.

\section{Summary and conclusions}

We surveyed the activity of the member galaxies in A983 and 1731 , focusing on their star formation and black hole accretion. Both clusters are located at $z \sim 0.2$ and their members were selected using spectroscopic redshifts. The total IR luminosity and $M_{*}$ of the members were computed using SED fitting. The total IR luminosity was used to compute the obscured SFR of the galaxies. Furthermore, the spectroscopic follow-up allowed us to obtain redshifts and independent unobscured SFR measurements, using the $\mathrm{H}_{\alpha}$ line flux. AGN were identified using an IR colour cut, narrow line flux ratio, and broad-line detection, and were removed from the SF analysis.

The distribution of passive population of galaxies presents a density peak in the central region for both clusters, albeit the number of passive objects is twice as great in A983 as in A1731. A clear difference emerged when we compared the 2D distribution of the star-forming galaxies: A983 presents a symmetric distribution of star-forming members, whereas those in A1731 were extended along what appeared to be a filamentlike structure. Higher values for the sSFR were found in both cluster outskirts, at $2-3 \mathrm{Mpc}\left(\sim 1-1.5 r_{200}\right)$ in clustercentric distance. A983 showed the typical characteristics of an evolved cluster. In contrast, A1731 appeared to be undergoing a phase of active accretion of field galaxies. The $50 \%$ of members hosted in the extended structure in A1731 presented lower values of the sSFR with respect to the other members at the same clustercentric distance. This suggested the possible effect of
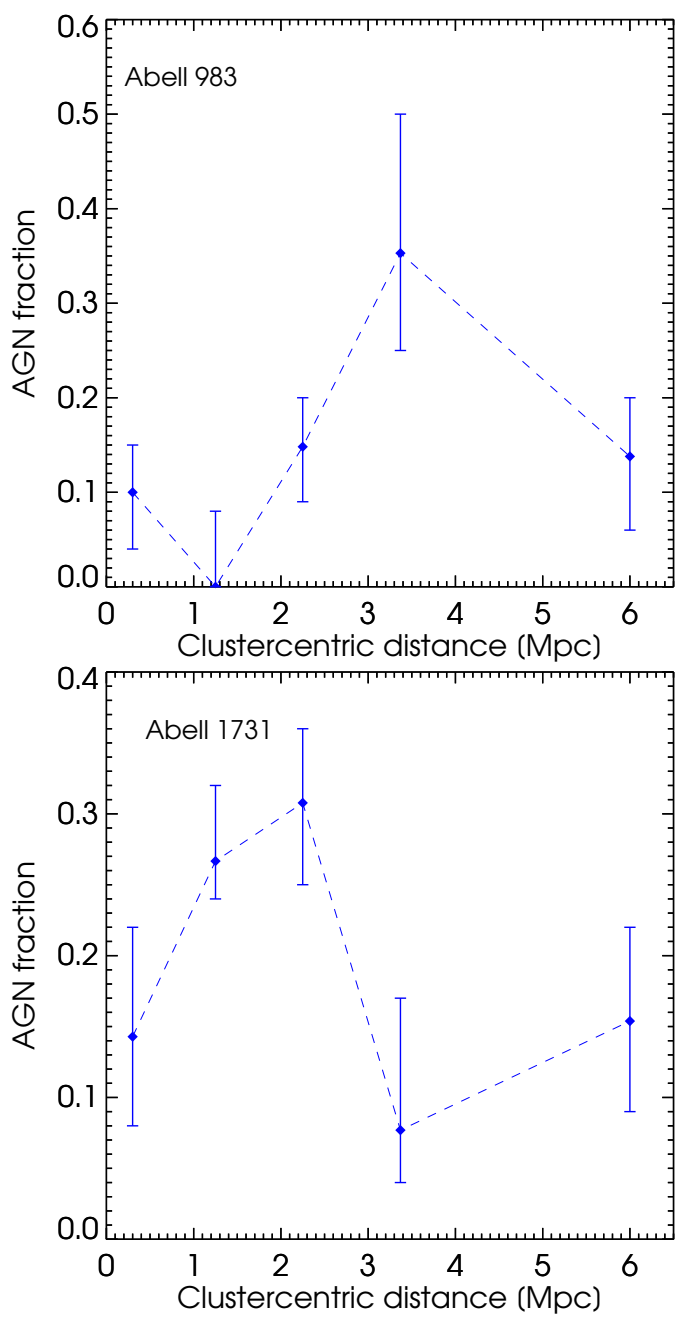

Fig. 15. Radially binned 1D distribution of the AGN fraction plotted against the clustercentric distance, for A983 (left panel) and A1731 (right panel). The errorbars are computed using the bootstrap method.

galaxy-galaxy interactions in reducing the sSFR, via harassment and subsequent suffocation, which was expected owing to the pre-processing of galaxies in the transitional environment, such as filaments or galaxy groups.

The activity of AGN depends on the availability of cold gas in the central regions of the galaxy, and on the dynamical processes that influence its accretion on the black hole. The diagnostics we used allowed us to observe a radial trend in the presence of AGN which were, on average, found at intermediate clustercentric distances $\left(\sim 3 \mathrm{Mpc}, \sim 1.5 r_{200}\right)$, where galaxy-galaxy interactions are more frequent (Balogh et al. 2004; Gallazzi et al. 2009). These results are in agreement with Haines et al. (2013) and Pimbblet et al. (2013). The cluster environment appears to have an influence on a dual scale: on a small scale, gas instabilities are promoted because of the frequent gravitational and hydrodynamical interactions, which lead to new SF and black hole accretion episodes; on the larger scale, further accretion of cold gas on the galaxies is blocked, suffocating the star formation and AGN activity. Further investigations, using both observations and simulations are necessary. Additional spectroscopic observations and X-ray imaging are required to unmistakably identify the presence of infalling groups or filaments in A1731. This would help to disentangle the effects of physical processes that are responsible for the accelerated ageing of cluster members. 
Acknowledgements. The authors would like to thank the anonymous referee whose suggestions improved the quality of the manuscript. The authors would also like to thank Tom Jarrett for kindly providing his package for the reduction of the WIRC near-IR data. Finally, the authors would like to thank Andrea Biviano for kindly providing their code (Mamon et al. 2010) for the cluster membership selection.

\section{References}

Ahn, C. P., Alexandroff, R., \& Allende Prieto, C., et al. 2014, ApJS, 211, 17 Baldwin, J. A., Phillips, M. M., \& Terlevich, R. 1981, PASP, 93, 5 Balogh, M. L., Schade, D., Morris, S. L., et al. 1998, ApJ, 504, L75 Balogh, M. L., Navarro, J. F., \& Morris, S. L. 2000, ApJ, 540, 113

Balogh, M. L., Baldry, I. K., Nichol, R., et al. 2004, ApJ, 615, L101

Bertin, E., \& Arnouts, S. 1996, A\&AS, 117, 393

Bertin, E., Mellier, Y., Radovich, M., et al. 2002, in Astronomical Data Analysis Software and Systems XI, eds. D. A. Bohlender, D. Durand, \& T. H. Handley, ASP Conf. Ser., 281, 228

Biviano, A., \& Katgert, P. 2004, A\&A, 424, 779

Biviano, A., Fadda, D., Durret, F., Edwards, L. O. V., \& Marleau, F. 2011, A\&A, 532, A77

Bruzual, G., \& Charlot, S. 2003, MNRAS, 344, 1000

Calzetti, D., Armus, L., Bohlin, R. C., et al. 2000, ApJ, 533, 682

Cohen, S. A., Hickox, R. C., Wegner, G. A., Einasto, M., \& Vennik, J. 2014, in Am. Astron. Soc. Meet. Abstr., 223, 358.06

Cohen, S. A., Hickox, R. C., \& Wegner, G. A. 2015, ApJ, 806, 85

da Cunha, E., Charlot, S., \& Elbaz, D. 2008, MNRAS, 388, 1595

De Lucia, G., Weinmann, S., Poggianti, B. M., Aragón-Salamanca, A., \& Zaritsky, D. 2012, MNRAS, 423, 1277

De Propris, R., Colless, M., \& Driver, S. P., et al. 2003, MNRAS, 342, 725

Diaferio, A., Kauffmann, G., Balogh, M. L., et al. 2001, MNRAS, 323, 999

Diolaiti, E., Bendinelli, O., Bonaccini, D., et al. 2000, in Astronomical Data Analysis Software and Systems IX, eds. N. Manset, C. Veillet, \& D. Crabtree, ASP Conf. Ser., 216, 623

Dressler, A. 1980, ApJ, 236, 351

Edwards, L. O. V., Fadda, D., Biviano, A., \& Marleau, F. R. 2010, AJ, 139, 434

Fadda, D., \& Duc, P.-A. 2002, in Tracing Cosmic Evolution with Galaxy Clusters, eds. S. Borgani, M. Mezzetti, \& R. Valdarnini, ASP Conf. Ser., 268, 297

Fadda, D., Girardi, M., Giuricin, G., Mardirossian, F., \& Mezzetti, M. 1996, ApJ, 473, 670

Fadda, D., Marleau, F. R., \& Storrie-Lombardi, L. J., et al. 2006, AJ, 131, 2859

Fadda, D., Biviano, A., Marleau, F. R., Storrie-Lombardi, L. J., \& Durret, F. 2008, ApJ, 672, L9
Fukugita, M., Shimasaku, K., \& Ichikawa, T. 1995, PASP, 107, 945 Gallazzi, A., Bell, E. F., \& Wolf, C., et al. 2009, ApJ, 690, 1883 Gunn, J. E., \& Gott, III, J. R. 1972, ApJ, 176, 1

Haines, C. P., Pereira, M. J., Smith, G. P., et al. 2013, ApJ, 775, 126 Haines, C. P., Pereira, M. J., Smith, G. P., et al. 2015, ApJ, 806, 101 Hao, L., Strauss, M. A., Fan, X., et al. 2005, AJ, 129, 1795

Hong, J., Im, M., Kim, M., \& Ho, L. C. 2015, ApJ, 804, 34 Kauffmann, G., \& Charlot, S. 1998, MNRAS, 297, L23

Kauffmann, G., Heckman, T. M., \& Tremonti, C., et al. 2003, MNRAS, 346, 1055

Kennicutt, Jr., R. C. 1998, ApJ, 498, 541

Kennicutt, Jr., R. C., Hao, C.-N., \& Calzetti, D., et al. 2009, ApJ, 703, 1672

Kewley, L. J., Dopita, M. A., Sutherland, R. S., Heisler, C. A., \& Trevena, J. 2001, ApJ, 556, 121

Kewley, L. J., Groves, B., Kauffmann, G., \& Heckman, T. 2006, MNRAS, 372, 961

Larson, R. B., Tinsley, B. M., \& Caldwell, C. N. 1980, ApJ, 237, 692

Lee, N., Le Floc'h, E., \& Sanders, D. P., et al. 2010, ApJ, 717, 175

Mamon, G. A., Biviano, A., \& Murante, G. 2010, A\&A, 520, A30

Marleau, F. R., Fadda, D., Appleton, P. N., et al. 2007, ApJ, 663, 218

McConnachie, A. W., Patton, D. R., Ellison, S. L., \& Simard, L. 2009, MNRAS, 395,255

Noeske, K. G., Faber, S. M., Weiner, B. J., et al. 2007, ApJ, 660, L47

Okamoto, T., \& Nagashima, M. 2003, ApJ, 587, 500

Oliver, S., Frost, M., \& Farrah, D., et al. 2010, MNRAS, 405, 2279

Osterbrock, D. E. 1989, Ann. NY Acad. Sci., 571, 99

Peng, Y., Maiolino, R., \& Cochrane, R. 2015, Nature, 521, 192

Petrosian, V. 1976, ApJ, 209, L1

Pimbblet, K. A., Shabala, S. S., Haines, C. P., Fraser-McKelvie, A., \& Floyd, D. J. E. 2013, MNRAS, 429, 1827

Sabater, J., Best, P. N., \& Argudo-Fernández, M. 2013, MNRAS, 430, 638

Skrutskie, M. F., Cutri, R. M., \& Stiening, R., et al. 2006, AJ, 131, 1163

Springel, V., White, S. D. M., Jenkins, A., et al., 2005, Nature, 435, 629

Steinhauser, D., Haider, M., Kapferer, W., \& Schindler, S. 2012, A\&A, 544, A54

Stern, D., Eisenhardt, P., \& Gorjian, V., et al. 2005, ApJ, 631, 163

Surace, J., Shupe, D. L., Farg, F., et al. 2005, BAAS, 1246, 37

van Dokkum, P. G. 2001, PASP, 113, 1420

Vogelsberger, M., Genel, S., Springel, V., et al. 2014, MNRAS, 444, 1518

Wetzel, A. R., Tinker, J. L., Conroy, C., \& van den Bosch, F. C. 2013, MNRAS, 432, 336

Wijesinghe, D. B., Hopkins, A. M., Brough, S., et al. 2012, MNRAS, 423, 3679

Wolf, C., Aragón-Salamanca, A., Balogh, M., et al. 2009, in The Starburst-AGN Connection, eds. W. Wang, Z. Yang, Z. Luo, \& Z. Chen, ASP Conf. Ser., 408, 248 